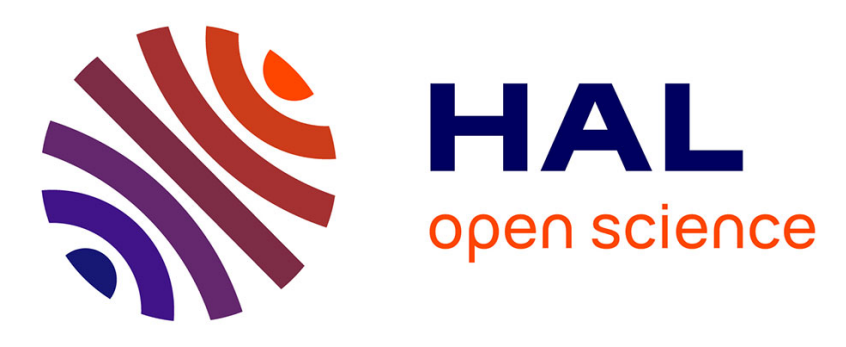

\title{
Proportional-delayed controllers design for LTI-systems a geometric approach
}

\author{
José-Enrique Hernández-Díez, César Fernando Méndez Barrios, Sabine \\ Mondié, Silviu-Iulian Niculescu, Emilio-Jorge González-Galván
}

\section{To cite this version:}

José-Enrique Hernández-Díez, César Fernando Méndez Barrios, Sabine Mondié, Silviu-Iulian Niculescu, Emilio-Jorge González-Galván. Proportional-delayed controllers design for LTIsystems a geometric approach. International Journal of Control, 2018, 91 (4), pp.907-925. 10.1080/00207179.2017.1299943 . hal-02281719

\section{HAL Id: hal-02281719}

https://hal-centralesupelec.archives-ouvertes.fr/hal-02281719

Submitted on 26 Mar 2021

HAL is a multi-disciplinary open access archive for the deposit and dissemination of scientific research documents, whether they are published or not. The documents may come from teaching and research institutions in France or abroad, or from public or private research centers.
L'archive ouverte pluridisciplinaire HAL, est destinée au dépôt et à la diffusion de documents scientifiques de niveau recherche, publiés ou non, émanant des établissements d'enseignement et de recherche français ou étrangers, des laboratoires publics ou privés. 
To appear in the International Journal of Control

Vol. 00, No. 00, Month 20XX, 1-28

\title{
Proportional-Delayed Controllers Design for LTI-Systems: A Geometric Approach
}

J.-E. Hernández-Díez ${ }^{\mathrm{a}}$, C.-F. Méndez-Barrios ${ }^{\mathrm{a}}$, S. Mondiéb ${ }^{\text {, S.-I. Niculescu }}{ }^{\mathrm{c}}$ and E.-J. González-Galván ${ }^{\mathrm{a}}$.

a Department of Electrical and Mechanical Engineering, Universidad Autónoma de San Luis Potosí (UASLP), 8, Dr. Manuel Nava, 78290, San Luis Potosí, MX; ${ }^{b}$ Department of Automatic Control CINVESTAV-IPN, Mexico City 14-740, Mexico; ${ }^{c}$ L2S (UMR CNRS 8506), CNRS-Supélec, 3, rue Joliot Curie, 91192, Gif-sur-Yvette, France.

(Received 00 Month 20XX; accepted 00 Month 20XX)

\begin{abstract}
This paper focuses on the design of $P-\delta$ controllers for single-input-single-output (SISO) linear timeinvariant (LTI) systems. The basis of this work is a geometric approach allowing to partitioning the parameter space in regions with constant number of unstable roots. This methodology defines the hyperplanes separating the aforementioned regions and characterizes the way in which the number of unstable roots changes when crossing such a hyper-plane. The main contribution of the paper is that it provides an explicit tool to find $P-\delta$ gains ensuring the stability of the closed-loop system. In addition, the proposed methodology allows to design a non-fragile controller with a desired exponential decay rate $\sigma$. Several numerical examples illustrate the results and a haptic experimental setup shows the effectiveness of $P-\delta$ controllers.
\end{abstract}

\section{Keywords: Time-Delay Systems, Proportional-Delayed Controllers, Stability, Fragility}

\section{Introduction}

Low-order controllers are one of the most widely applied strategies to control industrial processes (see, e.g.,(Aström \& Hägglund, 2001; Silva et al., 2005; O'Dwyer, 2009)). This "popularity" can be attributed to their particular distinct features: simplicity and easy implementation. Among these controllers, those of PID-type are known to be able to cope with uncertainties, disturbances, elimination of steady-state errors and transient response improvement (Aström \& Hägglund, 1995; Méndez-Barrios et al., 2008; Méndez-Barrios, 2011; Ramírez et al., 2016). The main drawbacks of PID controllers, reported in (Aström \& Hägglund, 1995; Altmann \& Macdonald, 2005), lies in the tuning of the derivative term, which may amplify high-frequency measurement noise. In fact, as mentioned in (Aström \& Hägglund, 2001; O'Dwyer, 2009; Smuts, 2011) the above arguments advise to avoid the derivative action in most applications.

The Euler approximation of the derivative,

$$
y^{\prime}(t) \approx \frac{y(t)-y(t-\epsilon)}{\epsilon},
$$

for small $\epsilon>0$, suggests to replace the derivative action by using delays (Suh \& Bien, 1980; Niculescu \& Michiels, 2004). It is well accepted that the presence of a delay in the feedback loop of continuous-time systems is often accompanied with oscillations and instability, bandwidth

${ }^{*}$ Corresponding author. Email: cerfranfer@gmail.com 
sensitivity, among others, see, for instance, (Niculescu, 2001; Michiels \& Niculescu, 2014). However, it is worth mentioning that there exist some situations when the delay may improve the system stability as explained in the classical example (Abdallah et al., 1993; Sipahi et al., 2011) where an oscillator is controlled by one delay "block": (gain, delay), with positive gains and small delay values (a detailed analysis of such an approach can be found in (Niculescu et al., 2010)). Inspired by the above observation, the design of low-order controllers with delay as a control parameter have been addressed in several works, for example, (Niculescu \& Michiels, 2004) (stabilizing chains of integrators by using delays), (Kharitonov et al., 2005) (multiple delay blocks), (Mazenc et al., 2003) (bounded input, single delay), to mention a few.

Recently, simple tuning rules for assigning the dominant poles of second-order systems in closedloop with a proportional-delayed control (called $P-\delta$ controller in this paper) were presented in (Villafuerte et al., 2013). Moreover, it was shown through an experimental setup that the $P-\delta$ controller outperforms standard proportional derivative controllers in terms of position error and control effort.

In this contribution, motivated by the above results on second order systems, as well as by the geometric ideas developed in (Morărescu \& Niculescu, 2007; Morărescu et al., 2011), we characterize the stability regions in the parameter space of a $P-\delta$ controller in closed-loop with general SISO systems. The approach consists in two steps:

(i) the construction of the stability crossing curves in the parameter space defined by the gains " $k_{p}$ " (proportional gain) and " $k_{\delta}$ " (a proportional gain related to a delay block with delay " $\tau$ "),

(ii) the explicit computation of the roots crossing direction (to stability or instability) which enables the determination of the number of unstable roots in each region.

In the above procedure, the first step sends back to the $\mathcal{D}$-decomposition method suggested by (Neimark, 1949) in the 40s (see (Gryazina, 2004; Gryazina et al., 2008; Michiels \& Niculescu, 2014) for further comments) or to the parameter space approach (see, for instance, (Bhattacharyya et al., 1995; Ackermann, 2002) or (Silva et al., 2005) and the references therein). In the sequel, the stability crossing curves represent the collection of all points for which the characteristic equation of the closed-loop system has at least one root on the imaginary axis. These boundaries define a "partition" of the space of parameters in several regions, each region having a constant number of unstable roots for all the parameters inside the region. In the second step, the use of some properties of analytic functions helps detecting if a region has more or fewer unstable roots than neighboring regions. This allows to find the regions with no unstable roots which corresponds to controller gains guaranteing the stability of the closed-loop system.

Although we use the general methodology introduced in (Méndez-Barrios, 2011) for PID-type controllers, we have to address a new problem which is revealed by the $P-\delta$ controller, namely, the case of even transfer functions that requires a special treatment. We also propose a characterization of multiple positive real roots that allows to discriminate some unstable regions of the space of parameters. In addition, we introduce a novel approach for the accounting of unstable roots. To complement the study, we discuss the performance of the closed-loop system in terms of $\sigma$-stabilization, as well as the controller fragility, which consists in finding the maximal parameter deviation guaranteing the closed-loop stability.

The paper is organized as follows: the problem formulation and the class of system under consideration are described in Section 2. The procedures to derive the stability crossing curves and root crossing directions are given in Section 3 and 4, respectively. Section 5 includes the $\sigma$-stability analysis as well as an algorithm to measure the fragility of a given $P-\delta$ controller. Section 6 presents several illustrative numerical examples and experimental results of the $P-\delta$ control of a hapticvirtual system. The paper ends with concluding remarks stated in Section 7 .

Throughout the paper the following standard notations are adopted: $\mathbb{C}$ (RHP, LHP) is the set of complex numbers (with strictly positive, and strictly negative real parts), and $j:=\sqrt{-1}$. For $z \in \mathbb{C}, \bar{z}, \Re(z)(\Im(z))$ define the complex conjugate, real (imaginary) part of $z . \mathbb{R}\left(\mathbb{R}_{+}\right.$and $\mathbb{R}_{-}$) 
denotes the set of real numbers (strictly positive, strictly negative). Let $\boldsymbol{x}, \boldsymbol{y} \in \mathbb{C}^{n}$, the scalar product is denoted by $\langle\boldsymbol{x}, \boldsymbol{y}\rangle=\boldsymbol{y}^{H} \boldsymbol{x}$, where $\boldsymbol{y}^{H}$ is the complex conjugate transpose of $\boldsymbol{y}$. Finally, $\lceil\cdot\rceil$ denotes the ceiling function, i.e., if $x \in \mathbb{R}$, then $\lceil x\rceil$ is the smallest integer greater than or equal to $x$.

\section{Problem formulation}

Consider the class of proper SISO open-loop systems given by the transfer function:

$$
G(s):=\frac{P(s)}{Q(s)}=c^{T}(s \boldsymbol{I}-\boldsymbol{A})^{-1} \boldsymbol{b},
$$

where $\left(\boldsymbol{A}, \boldsymbol{b}, \boldsymbol{c}^{T}\right)$ is a state-space representation of the open-loop system. $P$ and $Q$ are polynomials defined as:

$$
\begin{aligned}
& P(s):=p_{m} s^{m}+p_{m-1} s^{m-1}+\ldots+p_{1} s+p_{0}, \\
& Q(s):=q_{n} s^{n}+q_{n-1} s^{n-1}+\ldots+q_{1} s+q_{0}
\end{aligned}
$$

where $p_{m} \neq 0$ and $q_{n} \neq 0$, that are assumed to satisfy the following:

Assumption 1: Polynomials $P$ and $Q$ satisfy the following conditions:

(i) $\operatorname{deg} Q \geq \operatorname{deg} P$.

(ii) $P(s)$ and $Q(s)$ are coprime polynomials.

(iii) $|P(j \omega)|>0, \forall \omega \in \mathbb{R}$.

(iv) If $Q\left(j \omega^{*}\right)=0$, then $\left|Q^{\prime}\left(j \omega^{*}\right)\right|>0, \omega^{*} \in \mathbb{R}$.

It is clear that Assumption 1-(i) states that the system is causal. If Assumption 1-(ii) is not fulfilled, this implies that there exist a non constant common factor $c(s)$, such that $P(s)=c(s) \tilde{P}(s)$ and $Q(s)=c(s) \tilde{Q}(s)$. In such a case, choosing $c(s)$ to be of the highest possible degree, the analysis can be pursued if $c(s)$ is a Hurwitz polynomial, otherwise, the system will remain unstable independently of the control action. Finally, in order to simplify the presentation, (iii) and (iv) are made to avoid having multiple roots on the imaginary axis in $P$ and $Q$, respectively.

The problem considered in this paper can be summarized as follows:

Problem 1: Find explicit conditions on the parameters $\left(\tau, k_{p}, k_{\delta}\right)$ such that the $P-\delta$ controller:

$$
C(s)=k_{p}+k_{\delta} e^{-\tau s}
$$

asymptotically stabilizes the closed-loop plant described by the transfer function (1).

Problem 2: For a fixed delay value $\tau^{*} \in \mathbb{R}_{+}$, derive an appropriate $P$ - $\delta$ controller $\boldsymbol{k}^{*}:=\left[k_{p}^{*}, k_{\delta}^{*}\right]^{T} \in$ $\mathbb{R}^{2}$ and a positive value $d$ such that the controller (4) stabilizes system (1) for any $k_{p}$ and $k_{\delta}$, satisfying:

$$
\sqrt{\left(k_{p}-k_{p}^{*}\right)^{2}+\left(k_{\delta}-k_{\delta}^{*}\right)^{2}}<d
$$

From a geometric point of view, for a fixed $\tau^{*} \in \mathbb{R}_{+}$, we can define the collection of all controller gains $\boldsymbol{k} \in \mathbb{R}^{2}$ as points in the $k_{p^{-}} k_{\delta}$ parameters plane. Therefore, Problem 1-(i) can be stated as the task of finding at least one region in the $k_{p^{-}} k_{\delta}$ parameters-plane such that, for all $\boldsymbol{k}$-points 
inside this region, the characteristic equation of the closed-loop system has all of its roots in the LHP. A region of the $k_{p}-k_{\delta}$ parameters-plane with such a feature is defined as a stability region.

\section{Stability crossing curves}

In this section we characterize the stability crossing curves for the $P$ - $\delta$ controllers applied to general LTI systems and we present some significant results on multiple roots. Before that, we remind the basic notions of our analysis and we introduce some useful definitions.

As established in the Introduction, we are interested in finding the stability regions in the $\left(k_{p}, k_{\delta}, \tau\right)$-parameter space (or in the $\left(k_{p}, k_{\delta}\right)$-parameter space, when $\tau$ is fixed), of the closed-loop system described by the following characteristic equation:

$$
\Delta\left(s ; k_{p}, k_{\delta}, \tau\right):=P(s)\left(k_{p}+k_{\delta} e^{-\tau s}\right)+Q(s)=0 .
$$

Remark 1: An important property of the quasipolynomial $\Delta\left(s ; k_{p}, k_{\delta}, \tau\right)$ is that its zeros are continuous functions with respect to their parameters (see, for instance, (Michiels \& Niculescu, 2014; Datko, 1978) and references therein). In this vein, the number of roots in the RHP can change only when some zeros cross the imaginary axis.

Bearing the above observations in mind, let us consider the following definitions.

Definition 1 (Frequency crossing set): The frequency crossing set $\Omega \in \mathbb{R}$ is the set of all $\omega$ such that, there exist at least a triplet $\left(k_{p}, k_{\delta}, \tau\right)$ for which

$$
\Delta\left(j \omega ; k_{p}, k_{\delta}, \tau\right)=P(j \omega)\left(k_{p}+k_{\delta} e^{-j \tau \omega}\right)+Q(j \omega)=0 .
$$

Remark 2: It is clear that if we take the complex conjugate of (6), the following equality holds:

$$
Q(-j \omega)+P(-j \omega)\left(k_{p}+k_{\delta} e^{j \omega \tau}\right)=\overline{Q(j \omega)+P(j \omega)\left(k_{p}+k_{\delta} e^{-j \omega \tau}\right)} .
$$

Therefore, in the rest of the paper we will consider only nonnegative frequencies, i.e., $\Omega \subset \mathbb{R}_{+} \cup\{0\}$.

Definition 2 (Stability Crossing Curves): The stability crossing curves $\mathcal{T}$ is the set of all parameters $\left(k_{p}, k_{\delta}, \tau\right) \in \mathbb{R}^{2} \times \mathbb{R}_{+}$for which there exist at least one $\omega \in \mathbb{R}_{+} \cup\{0\}$ such that $\Delta\left(j \omega ; k_{p}, k_{\delta}, \tau\right)=0$. For a fixed delay value $\tau^{*} \in \mathbb{R}_{+}$, any point $\boldsymbol{k} \in \mathcal{T}$ is known as a crossing point.

Remark 3: For analysis purpose, we consider in some situations $\tau^{*}$ as a fixed parameter. In such cases $\mathcal{T}$ will be composed by the parameters $\left(k_{p}, k_{\delta}, \tau^{*}\right) \in \mathbb{R}^{2} \times \mathbb{R}_{+}$satisfying Definition 2 .

\subsection{Stability crossing curves characterization}

In the derivation of the pure imaginary crossing curves for $P-\delta$ controllers a special situation arises when the open-loop transfer function $G$ is even ${ }^{1}$. In order to treat properly this scenario, we present a new result concerning the even case. The treatment for the cases $s=0$ and $s=j \omega$, $\omega \rightarrow \infty$ (neutral case) do not need such considerations.

\footnotetext{
${ }^{1}$ Although $G$ is a complex function, here we consider even functions in the real domain, i.e., $G(x)=G(-x), \forall x \in \mathbb{R}$.
} 


\subsubsection{Purely imaginary crossing curves}

Proposition 1 (Crossing curves characterization): Assume that $G$ is not an even function such that $\operatorname{deg} Q>\operatorname{deg} P$, and let $\tau \in \mathbb{R}_{+}$be a fixed value and $\Omega:=\bigcup_{\ell} \Omega_{\ell}$ for $\ell \in \mathbb{N}$, where the subsets $\Omega_{\ell}$ are defined as:

$$
\Omega_{\ell}:=\left\{\omega \in \mathbb{R}_{+} \mid \omega \in\left(\frac{\pi}{\tau}(\ell-1), \frac{\pi}{\tau} \ell\right)\right\} .
$$

Then, $\omega \in \Omega$ is a crossing frequency if and only if $\boldsymbol{k}(\omega):=\left[k_{p}(\omega), k_{\delta}(\omega)\right]^{T}$, where:

$$
\begin{aligned}
& k_{p}(\omega)=-\Re\left[\frac{Q(j \omega)}{P(j \omega)}\right]-\cot (\tau \omega) \Im\left[\frac{Q(j \omega)}{P(j \omega)}\right], \\
& k_{\delta}(\omega)=\csc (\tau \omega) \Im\left[\frac{Q(j \omega)}{P(j \omega)}\right],
\end{aligned}
$$

defines a crossing point $\boldsymbol{k}(\omega) \in \mathcal{T}$.

Proof. Consider the characteristic equation (5). It is clear that all the crossing points $\boldsymbol{k} \in \mathcal{T}$ are given by the pairs $\boldsymbol{k} \in \mathbb{R}^{2}$ solving (5) for $s=j \omega$. Taking the real and imaginary part gives the following:

$$
\begin{aligned}
& \Re\left[\Delta\left(j \omega ; k_{p}, k_{\delta}, \tau\right)\right]=0, \\
& \Im\left[\Delta\left(j \omega ; k_{p}, k_{\delta}, \tau\right)\right]=0 .
\end{aligned}
$$

The solution of this system for $k_{p}$ and $k_{\delta}$ leads to (8) and (9) by using simple algebraic manipulations. Furthermore, from (8) and (9), it can be observed that $\boldsymbol{k}(\omega)$ is a real solution if and only if $\omega \neq n \frac{\pi}{\tau}$ for $n \in \mathbb{Z}$. Therefore, $\boldsymbol{k}(\omega)$ is a real solution for all $\omega \in \Omega$.

In the previous result we have assumed that $G$ was not an even function. Now, we focuses on the particular case where $G$ is even.

Proposition 2: Let $\tau \in \mathbb{R}_{+}$be a fixed value and $G(s)$ an even function. Then the set of all stability crossing curves is composed by the following set of lines:

(i)

$$
\begin{aligned}
& k_{p}=-G^{-1}(j \omega), \quad \omega \in \mathbb{R}_{+} \cup\{0\} \\
& k_{\delta}=0
\end{aligned}
$$

(ii)

$$
k_{p}+(-1)^{\ell} k_{\delta}=-G^{-1}(j \omega), \quad \omega=\frac{\ell \pi}{\tau}, \ell \in \mathbb{N} .
$$

Proof. Assume that $\omega$ is a crossing frequency. Then, from (5), the following equalities must hold:

$$
\begin{aligned}
\Delta\left(j \omega ; k_{p}, k_{\delta}, \tau\right) & =0, \\
G^{-1}(j \omega)+\left(k_{p}+k_{\delta} e^{-j \omega \tau}\right) & =0 .
\end{aligned}
$$

Now, since $G$ is even by hypothesis, $G^{-1}$ is also an even function and we have:

$$
\Re\left[G^{-1}(j \omega)\right]=G^{-1}(j \omega) \quad \text { and } \quad \Im\left[G^{-1}(j \omega)\right]=0 .
$$


From the last expression one can notice that $\omega$ is a crossing frequency if and only if:

$$
\Im\left[\Delta\left(j \omega ; k_{p}, k_{\delta}, \tau\right)\right]=0 \quad \Rightarrow \quad k_{\delta} \sin (\omega \tau)=0 .
$$

The solutions of (14) implies (12) and (13).

\subsubsection{Real crossing curves and neutral type condition}

Proposition 3: Let $\tau \in \mathbb{R}_{+}$be a fixed value. Then, the line:

$$
k_{\delta}=-k_{p}-\frac{q_{0}}{p_{0}},
$$

represents a stability crossing curve. Furthermore, this corresponds to a crossing through the origin of the complex plane.

Proof. By taking $s=0$ in (5), we get:

$$
\begin{aligned}
\Delta\left(0 ; k_{p}, k_{\delta}, \tau\right) & =0, \\
\Leftrightarrow q_{0}+p_{0}\left(k_{p}+k_{\delta}\right) & =0,
\end{aligned}
$$

and (15) follows straightforwardly.

When $\operatorname{deg} Q=\operatorname{deg} P$ the system is of neutral-type. The behaviour of the neutral root chain far from the origin (see, for instance, (Krall, 1964; Hale \& Verduyn Lunel, 1993)) imposes necessary stability conditions on $k_{p}$ and $k_{d}$.

Proposition 4 (Neutral-Type Condition): Assume that $\operatorname{deg} Q=\operatorname{deg} P$. Then, if $\boldsymbol{k} \in \mathbb{R}^{2}$ is a stabilizing controller, the following condition holds:

$$
\left|k_{\delta}\right|<\left|k_{p}+\frac{q_{n}}{p_{n}}\right|
$$

Furthermore, this inequality defines a stability crossing curve.

Proof. The equation (5) can be rewritten as:

$$
e^{\tau s}=-\frac{k_{\delta} P(s)}{k_{p} P(s)+Q(s)} .
$$

Assuming that $p_{n} \neq 0$ and considering the existence of a solution of this equation as $s \rightarrow \infty$ leads to the following expression:

$$
e^{\tau s}=-\frac{k_{\delta} p_{n}}{k_{p} p_{n}+q_{n}}+\mathcal{O}\left(\frac{1}{s}\right) .
$$

From (18), we have that the real part of this solution behaves as:

$$
\Re\{s\}=\frac{1}{\tau} \log \left|\frac{k_{\delta} p_{n}}{k_{p} p_{n}+q_{n}}\right| .
$$

By taking into account the above expression, one can see that if condition (16) is not satisfied, there is at least one root of (5) with positive real part. Therefore, this is a necessary condition for $\boldsymbol{k}$ to be a stabilizing controller pair. 
We present next a summary of the results of this section. Given all stability crossing points $\boldsymbol{k}(\omega)$ and the frequency crossing set $\Omega$, we can define each stability crossing curve through its continuity, as follows:

$$
\begin{aligned}
& \mathcal{T}_{0}:=\left\{\boldsymbol{k} \in \mathbb{R}^{2} \mid k_{\delta}=-k_{p}-\frac{q_{0}}{p_{0}}\right\}, \\
& \mathcal{T}_{i}:=\left\{\boldsymbol{k}(\omega) \in \mathbb{R}^{2} \mid \omega \in \Omega_{i} \text { for } i \in \mathbb{N}\right\} .
\end{aligned}
$$

For the special case of even transfer functions the stability crossing curves also include:

$$
\mathcal{T}_{p_{\ell}}:=\left\{\boldsymbol{k} \in \mathbb{R}^{2} \mid k_{\delta}=(-1)^{\ell+1}\left\{k_{p}+\frac{Q\left(j \omega_{\ell}\right)}{P\left(j \omega_{\ell}\right)}\right\}\right\}, \quad \text { for } \ell \in \mathbb{N} .
$$

By defining $\mathcal{T}_{p_{\ell}}:=\emptyset$ when $G$ is not even, we can describe the set $\mathcal{T}$ as:

$$
\mathcal{T}=\bigcup_{i} \mathcal{T}_{i} \bigcup_{\ell} \mathcal{T}_{p_{\ell}}, \quad i \in \mathbb{N} \cup\{0\}, \ell \in \mathbb{N}
$$

\subsection{Multiple roots characterization}

In this section we present two results concerning multiple roots. The first one, concerns positive real roots while the second one gives a necessary condition to characterize multiple pure imaginary roots.

Proposition 5: Let $\tau \in \mathbb{R}_{+}$be a fixed value, and $\boldsymbol{k} \in \mathbb{R}^{2}$. Then, if $\boldsymbol{k}$ is a stabilizing controller the following condition holds:

$$
\boldsymbol{k} \neq \tilde{\boldsymbol{k}}(\tilde{s}):=\left[\tilde{k}_{p}(\tilde{s}), \tilde{k}_{\delta}(\tilde{s})\right]^{T}
$$

for all $\tilde{s} \in \mathbb{R}_{+}$such that $P(\tilde{s}) \neq 0$, with $\tilde{k}_{p}(\tilde{s})$ and $\tilde{k}_{\delta}(\tilde{s})$ defined as follows:

$$
\begin{aligned}
& \tilde{k}_{p}(\tilde{s}):=-\frac{Q(\tilde{s})}{P(\tilde{s})}-\frac{1}{\tau} \frac{P(\tilde{s}) Q^{\prime}(\tilde{s})-P^{\prime}(\tilde{s}) Q(\tilde{s})}{P^{2}(\tilde{s})}, \\
& \tilde{k}_{\delta}(\tilde{s}):=\frac{1}{\tau} \frac{P(\tilde{s}) Q^{\prime}(\tilde{s})-P^{\prime}(\tilde{s}) Q(\tilde{s})}{P^{2}(\tilde{s})} e^{\tau \tilde{s}}
\end{aligned}
$$

where $P^{\prime}$ and $Q^{\prime}$ denote the derivative of $P$ and $Q$, respectively. Furthermore, if $\boldsymbol{k}=\tilde{\boldsymbol{k}}(\tilde{s})$ for some $\tilde{s} \in \mathbb{R}_{+}$, then the characteristic equation of the closed-loop system has at least two roots in the RHP of the complex plane at $s=\tilde{s}$. Furthermore, we define the curve:

$$
\mathcal{T}_{u}:=\left\{\boldsymbol{k} \in \mathbb{R}^{2} \mid \boldsymbol{k}=\tilde{k}(\tilde{s}), \forall \tilde{s}>0\right\}
$$

Proof. The characteristic equation (5) can be rewritten as follows:

$$
k_{p}+k_{\delta} e^{-\tau s}+\frac{Q(s)}{P(s)}=0,
$$


and its derivative is computed as:

$$
-\tau k_{\delta} e^{-\tau s}+\frac{P(s) Q^{\prime}(s)-P(s)^{\prime} Q(s)}{P^{2}(s)}=0 .
$$

Hence, if both equations have a solution $s=\tilde{s}$ for some $\tilde{s} \in \mathbb{R}_{+}$, then the characteristic equation (5) has a multiple root with at least multiplicity two at $s=\tilde{s}$. Clearly, the closed-loop system with such roots in its characteristic equation is an unstable system. Finally, by solving (27)-(28) with respect to $k_{p}$ and $k_{\delta}$, we obtain the conditions (25) and (26), respectively.

The next result deals with multiple pure imaginary roots characterization:

Proposition 6: Let $\tau \in \mathbb{R}_{+}$be a fixed value, $\boldsymbol{k} \in \mathbb{R}^{2}$ and let $\Omega_{m} \subset \Omega$ be the subset of crossing frequencies with multiplicity $m \geq 2$. Assume that there is no constant $a \in \mathbb{R}_{+}$satisfying the following condition:

$$
\frac{\Im\{f(j \omega)\}}{\Re\left\{f^{\prime}(j \omega)\right\}}=a, \quad \forall \omega \in \mathbb{R}
$$

where $f(j \omega):=G^{-1}(j \omega)$. Then, the cardinality of $\Omega_{m}$, i.e., $\left|\Omega_{m}\right|$, is finite. Furthermore, if $\Omega_{m} \neq \emptyset$ and there exist some constant $a \in \mathbb{R}_{+}$fulfilling (29), then the cardinality of $\Omega_{m}$ is infinite (but countable) for some $m \geq 2$.

Proof. First, assume that there is no constant $a \in \mathbb{R}_{+}$, that satisfies condition (29). Now, observe that for some $m \geq 2$ it is possible that $\Omega_{m}$ is an empty set. In such a situation, by definition, $\left|\Omega_{m}\right|=0$, and the result is valid.

Consider now that $\omega^{*} \in \Omega_{m} \neq \emptyset$, for some $m \geq 2$. By hypothesis there exist $\boldsymbol{k} \in \mathbb{R}^{2}$, such that:

$$
\begin{aligned}
\Delta\left(j \omega^{*} ; k_{p}, k_{\delta}, \tau\right) & =0, \\
\Rightarrow f\left(j \omega^{*}\right)+\left(k_{p}+k_{\delta} e^{-j \omega^{*} \tau}\right) & =0 .
\end{aligned}
$$

Thus, since $j \omega^{*}$ is a root with multiplicity $m \geq 2$, the following equality must hold:

$$
f^{\prime}\left(j \omega^{*}\right)-j k_{\delta} \tau e^{-j \omega^{*} \tau}=0 .
$$

From (30b) and (31) we have that,

$$
\begin{aligned}
k_{p} & =-f\left(j \omega^{*}\right)+\frac{j}{\tau} f^{\prime}\left(j \omega^{*}\right), \\
k_{\delta} & =-\frac{j}{\tau} e^{j \omega^{*} \tau} f^{\prime}\left(j \omega^{*}\right) .
\end{aligned}
$$

Now, from the fact that both $k_{p}$ and $k_{\delta}$ are real numbers, we have that $j \omega^{*}$ is a multiple solution if the following statements hold simultaneously:

$$
\begin{aligned}
-\Im\left\{f\left(j \omega^{*}\right)\right\}+\frac{1}{\tau} \Re\left\{f^{\prime}\left(j \omega^{*}\right)\right\} & =0, \\
\Re\left\{e^{j \omega^{*} \tau} f^{\prime}\left(j \omega^{*}\right)\right\} & =0 .
\end{aligned}
$$

Next, we know by assumption that there is no constant $a \in \mathbb{R}_{+}$fulfilling (29). This implies that there is no constant delay $\tau \in \mathbb{R}_{+}$nullifying (34) for all $\omega \in \mathbb{R}$, and consequently for a fixed delay $\tau \in \mathbb{R}_{+}$, condition (34) is of polynomial-type, impliying a finite number of solutions. As both 
(34)-(35) must be fulfilled simultaneously, we conclude that $\Omega_{m}$ has a finite number of elements. Furthermore, taking into account the previous arguments, we conclude that if there exists some $a \in \mathbb{R}_{+}$satisfying (29), then for $\tau=1 / a$, condition (34) will be fulfilled for all $\omega \in \mathbb{R}$, and since (35) has an infinite (but countable) number of solutions, this implies that $\Omega_{m}$ has an infinite but countable number of elements. The proof is complete by noticing that for $m_{1}, m_{2} \in \mathbb{N}$ with $m_{1}<m_{2}$, therefore $\Omega_{m_{1}} \supseteq \Omega_{m_{2}}$.

Remark 4: From the proof of Proposition 6, it is evident that (34)-(35) are necessary conditions for $j \omega^{*}$ to be a crossing frequency with multiplicity $m \geq 2$. Furthermore, if $m=2$, thus (34)-(35) are also a sufficient condition.

\section{Stability regions}

The results presented through Propositions 1-2 allow us to determine the values of $k_{p}$ and $k_{\delta}$ for which crossing roots exist, but do not give any information on their crossing direction. In order to characterize regions according their number of unstable roots, we must make a distinction between switches (crossing towards instability) and reversals (crossing towards stability), and carry out a careful accounting of the unstable roots in each region.

\subsection{Crossing directions characterization}

To determine the roots tendency as the vector $\boldsymbol{k}$ deviates from the curve $\mathcal{T}$, we start by introducing some notions. A stability crossing curve $\mathcal{T}_{i}$ for $i \neq 0$ is generated by $\boldsymbol{k}(\omega)$ for all $\omega \in \Omega_{i}$, therefore we define as positive direction of $\mathcal{T}_{i}$ the direction of the curve $\boldsymbol{k}(\omega)$ that corresponds to increasing $\omega$ 's. Fig. 1 illustrates a positive direction when $\omega$ increase from $\frac{\pi}{\tau}(i-1)$ to $\frac{\pi}{\tau} i$.

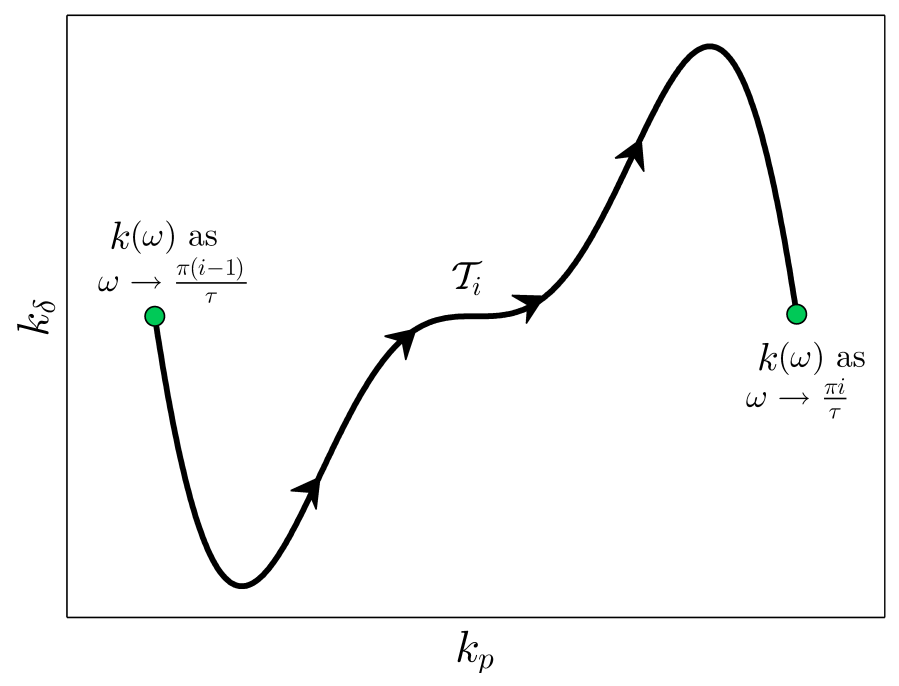

Figure 1. Positive direction of $\mathcal{T}_{i}$.

Remark 5: Recall the geometric approach of the basis orientation in $\mathbb{R}^{2}$ from linear algebra (Cullen, 1990). Let $\boldsymbol{u}:=\left[u_{1}, u_{2}\right]^{T}$ and $\boldsymbol{v}:=\left[v_{1}, v_{2}\right]^{T}$ be vectors of $\mathbb{R}^{2}$, and $\mathcal{B}$ be a basis on $\mathbb{R}^{2}$ on the field $\mathbb{R}$, defined as:

$$
\mathcal{B}:=\{\boldsymbol{u}, \boldsymbol{v}\}=\left\{\left[u_{1}, u_{2}\right]^{T},\left[v_{1}, v_{2}\right]^{T}\right\}
$$


$\mathcal{B}$ is said to be positively oriented if the shortest path from $\boldsymbol{u}$ to $\boldsymbol{v}$ is in the counterclockwise direction, and is said to be negatively oriented if the shortest path from $\boldsymbol{u}$ to $\boldsymbol{v}$ is in the clockwise direction. Then, $\mathcal{B}$ is positively oriented if and only if the following condition holds:

$$
\operatorname{det}[\boldsymbol{u} \boldsymbol{v}]=u_{1} v_{2}-u_{2} v_{1}>0 .
$$

Furthermore, $\mathcal{B}$ is negatively oriented if and only if the inequality (37) is reversed.

Proposition 7: Let $\tau \in \mathbb{R}_{+}$be a fixed delay, and consider the set of all stability crossing curves $\mathcal{T}_{i}, \forall i \in \mathbb{N}$. Then a pair of roots of (5) crosses from the LHP to the RHP of the complex plane as $\boldsymbol{k}$ traverses a stability crossing curve $\mathcal{T}_{i}$ from left to right with respect to the positive direction of $\mathcal{T}_{i}$ if $i$ is even. Furthermore, the crossing is reversed if $i$ is odd.

Proof. The characteristic equation (5) can be rewritten as:

$$
k_{p}+k_{\delta} e^{-\tau s}+G^{-1}(j \omega)=0 .
$$

Now, expressing $s=\sigma+j \omega$ leads to the following equation:

$$
u\left(k_{p}, k_{\delta}, \sigma, \omega\right)+j v\left(k_{\delta}, \sigma, \omega\right)=0,
$$

where:

$$
\begin{gathered}
u\left(k_{p}, k_{\delta}, \sigma, \omega\right):=k_{p}+k_{\delta} e^{-\tau \sigma} \cos (\tau \omega)+\Re\left[\frac{Q(\sigma+j \omega)}{P(\sigma+j \omega)}\right], \\
v\left(k_{\delta}, \sigma, \omega\right):=\quad-k_{\delta} e^{-\tau \sigma} \sin (\tau \omega)+\Im\left[\frac{Q(\sigma+j \omega)}{P(\sigma+j \omega)}\right] .
\end{gathered}
$$

Now, in order to fulfill (39), we have from (40) that the following transformation connects the complex plane $s$, with the parameter space $\boldsymbol{k}$ :

$$
\left[\begin{array}{l}
k_{p} \\
k_{\delta}
\end{array}\right]=-\left[\begin{array}{cc}
1 & e^{-\tau \sigma} \cos (\tau \sigma) \\
0 & -e^{-\tau \sigma} \sin (\tau \sigma)
\end{array}\right]^{-1}\left[\begin{array}{l}
\Re\left[\frac{Q(\sigma+j \omega)}{P(\sigma+j \omega)}\right] \\
\Im\left[\frac{Q(\sigma+j \omega)}{P(\sigma+j \omega)}\right]
\end{array}\right] .
$$

In order to analyze the conservation of the orientation of the map (41), we consider the total derivatives of $u$ and $v$, which are given by

$$
\begin{aligned}
d k_{p}+e^{-\tau \sigma} \cos (\tau \omega) d k_{\delta}+\frac{\partial u}{\partial \sigma} d \sigma+\frac{\partial u}{\partial \omega} d \omega & =0 \\
-e^{-\tau \sigma} \sin (\tau \omega) d k_{\delta}+\frac{\partial v}{\partial \sigma} d \sigma+\frac{\partial v}{\partial \omega} d \omega & =0
\end{aligned}
$$

respectively. Now, from the Cauchy-Reimann conditions (see, for instance, (Levinson \& Redheffer, 1970)) we known that:

$$
\frac{\partial u}{\partial \sigma}=\frac{\partial v}{\partial \omega}, \quad \text { and } \quad \frac{\partial u}{\partial \omega}=-\frac{\partial v}{\partial \sigma} .
$$


Applying (44) to (42) and (43), we get:

$$
\left[\begin{array}{l}
d k_{p} \\
d k_{\delta}
\end{array}\right]=-\frac{1}{\mathcal{D}}\left[\begin{array}{cc}
-e^{\tau \sigma} \sin (\tau \omega) & \left.-e^{-\tau \sigma} \cos (\tau \omega)\right) \\
0 & 1
\end{array}\right]\left[\begin{array}{cc}
\frac{\partial u}{\partial \sigma} & \frac{\partial u}{\partial \omega} \\
-\frac{\partial u}{\partial \omega} & \frac{\partial u}{\partial \sigma}
\end{array}\right]\left[\begin{array}{l}
d \sigma \\
d \omega
\end{array}\right]
$$

where $\mathcal{D}:=-e^{-\tau \sigma} \sin (\tau \omega)$. Finally, the jacobian transformation can be derived from (45) as:

$$
\frac{\partial\left(k_{p}, k_{\delta}\right)}{\partial(\sigma, \omega)}=\frac{1}{\mathcal{D}}\left[\left(\frac{\partial u}{\partial \sigma}\right)^{2}+\left(\frac{\partial u}{\partial \omega}\right)^{2}\right]
$$

where it is clear to see that:

$$
\operatorname{sgn}\left\{\frac{\partial\left(k_{p}, k_{\delta}\right)}{\partial(\sigma, \omega)}\right\}=\operatorname{sgn}\{\mathcal{D}\}
$$

According to Remark 5, the transformation (41) conserves the orientation if the sign of $\mathcal{D}$ is positive, and reverses the orientation if $\mathcal{D}<0$. Therefore, when $\boldsymbol{k}$ crosses the curve $\mathcal{T}_{i}$ from left to right with respect to the positive direction, a pair of roots of (5) crosses from the LHP to the RHP of the complex plane if $\mathcal{D}>0$, the crossing is from the RHP to the LHP if $\mathcal{D}<0$. Finally, notice that $\mathcal{D}$ is positive for all $\omega \in \Omega_{i}$ with $i$ even, and negative for all $\omega \in \Omega_{i}$ with $i$ odd.

Observe that Proposition 7 does not give any information about the crossing when $i=0$. The following result fills this gap.

Proposition 8: Given a fixed delay $\tau \in \mathbb{R}_{+}$. Then, one root of (5) crosses from the LHP to the RHP of the complex plane through the origin as $\boldsymbol{k}$ crosses $\mathcal{T}_{0}$ from left to right if the intersection of $\boldsymbol{k}$ and $\mathcal{T}_{0}$ is located at the left of the point $\boldsymbol{k}_{0} \in \mathcal{T}_{0}$, defined by:

$$
\boldsymbol{k}_{0}:=\left[k_{p_{0}}, k_{\delta_{0}}\right]^{T}=\left[\frac{p_{1} q_{0}-\left(\tau q_{0}+q_{1}\right) p_{0}}{\tau p_{0}^{2}}, \frac{p_{0} q_{1}-q_{0} p_{1}}{\tau p_{0}^{2}}\right]^{T} .
$$

Furthermore, the crossing of the root is from the RHP to the LHP if the intersection is located at the right of $\boldsymbol{k}_{0}$.

Proof. Let $s \in \mathbb{C}$ be a solution of (5), then the following equality holds:

$$
\Delta_{r}\left(s ; k_{p}, k_{\delta}, \tau\right):=\frac{1}{P(s)} \Delta\left(s ; k_{p}, k_{\delta}, \tau\right)=k_{p}+k_{\delta} e^{-\tau s}+\frac{Q(s)}{P(s)}=0,
$$

Now, according to the implicit function theorem (see, for instance, (Guggenheimer, 1977)), we known that:

$$
\frac{d s}{d k_{p}}=-\frac{\frac{\partial \Delta_{r}}{\partial k_{p}}}{\frac{\partial \Delta_{r}}{\partial s}}, \quad \frac{d s}{d k_{\delta}}=-\frac{\frac{\partial \Delta_{r}}{\partial k_{\delta}}}{\frac{\partial \Delta_{r}}{\partial s}}
$$

where:

$$
\frac{\partial \Delta_{r}}{\partial s}=-\tau k_{\delta} e^{-\tau s}+\frac{P(s) Q^{\prime}(s)-Q(s) P^{\prime}(s)}{P(s)^{2}}
$$




$$
\frac{\partial \Delta_{r}}{\partial k_{p}}=1, \quad \frac{\partial \Delta_{r}}{\partial k_{\delta}}=e^{-\tau s}
$$

Taking $s=0$, we get:

$$
\mathcal{S}\left(k_{\delta}\right):=\Re\left[\left.\frac{d s}{d k_{p}}\right|_{s=0}\right]=\Re\left[\left.\frac{d s}{d k_{\delta}}\right|_{s=0}\right]=\frac{p_{0}^{2}}{p_{0}^{2} \tau k_{\delta}+q_{0} p_{1}-p_{0} q_{1}} .
$$

Therefore, as $\boldsymbol{k}$ crosses in any direction from left to right of $\mathcal{T}_{0}$, one root of $(5)$ crosses from the LHP to the RHP of the complex plane through the origin if $\mathcal{S}\left(k_{\delta}\right)>0$, which implies that $k_{\delta}>k_{\delta_{0}}$. Furthermore, the crossing is from the RHP to the LHP if $\mathcal{S}\left(k_{\delta}\right)<0$, equivalent to $k_{\delta}>k_{\delta_{0}}$. Finally, by simple algebraic manipulations, one can see that the condition $k_{\delta}>k_{r_{0}}$ implies that $k_{p}<k_{p_{0}}$, as stated in Proposition 8.

As in the case of stability crossing curves, additional considerations must be taken into account when $G$ is an even function. Such a situation is considered below:

Proposition 9: Let $\tau \in \mathbb{R}_{+}$be a fixed delay and $G$ an even function. Then, as $\boldsymbol{k}$ crosses in any direction from the left side to the right side of $\mathcal{T}_{\ell}, \ell \in \mathbb{N}$ through the point $\hat{\boldsymbol{k}}:=\left[\hat{k}_{p}, \hat{k}_{\delta}\right]^{T} \in \mathcal{T}_{\ell}$, a pair of roots of (5) crosses from the LHP to the RHP of the complex plane, if $\hat{\boldsymbol{k}}$ satisfies the following conditions:

$$
\hat{k}_{\delta}>0 \text { for } \ell \text { even, } \quad \hat{k}_{\delta}<0 \text { for } \ell \text { odd }
$$

Furthermore, the crossing of the roots is from the RHP to the LHP if these inequalities are reversed.

Proof. The characteristic equation (5) can be reformulated as:

$$
\hat{\Delta}:=k_{p}+k_{\delta} e^{-\tau s}+G^{-1}(j \omega)=0 .
$$

Now, by the implicit function theorem, we get:

$$
\frac{d s}{d k_{p}}=-\frac{\frac{\partial \hat{\Delta}}{\partial k_{p}}}{\frac{\partial \hat{\Delta}}{\partial s}}, \quad \frac{d s}{d k_{\delta}}=-\frac{\frac{\partial \hat{\Delta}}{\partial k_{\delta}}}{\frac{\partial \hat{\Delta}}{\partial s}},
$$

where:

$$
\begin{gathered}
\frac{\partial \hat{\Delta}}{\partial s}=-\tau k_{\delta} e^{-\tau s}+\frac{P(s) Q^{\prime}(s)-Q(s) P^{\prime}(s)}{P(s)^{2}}, \\
\frac{\partial \hat{\Delta}}{\partial k_{p}}=1, \quad \frac{\partial \Delta_{r}}{\partial k_{\delta}}=e^{-\tau s} .
\end{gathered}
$$

Then, taking $s=j \omega_{\ell}$ leads to the following:

$$
\left.\frac{d s}{d k_{p}}\right|_{s=j \omega_{\ell}}=\frac{P^{2}\left(j \omega_{\ell}\right)}{\tau k_{\delta} P^{2}\left(j \omega_{\ell}\right)(-1)^{\ell}+P^{\prime}\left(j \omega_{\ell}\right) Q\left(j \omega_{\ell}\right)-P\left(j \omega_{\ell}\right) Q^{\prime}\left(j \omega_{\ell}\right)} .
$$

As $P$ and $Q$ are polynomials with only even powers of $s, P^{\prime}(s)$ and $Q^{\prime}(s)$ are polynomials with only odds powers of $s$. Then, $P\left(j \omega_{\ell}\right)$ and $Q\left(j \omega_{\ell}\right)$ are real-valued functions, moreover $P^{\prime}\left(j \omega_{\ell}\right)$ and 
$Q^{\prime}\left(j \omega_{\ell}\right)$ are purely imaginary functions. Using these facts and noticing that $\frac{d s}{d k_{\delta}}=\frac{d s}{d k_{\delta}} e^{-\tau s}$, we conclude that:

$$
\operatorname{sgn}\left\{\Re\left[\left.\frac{d s}{d k_{p}}\right|_{s=j \omega_{\ell}}\right]\right\}=\operatorname{sgn}\left\{(-1)^{\ell} k_{\delta}\right\}, \quad \operatorname{sgn}\left\{\Re\left[\left.\frac{d s}{d k_{\delta}}\right|_{s=j \omega_{\ell}}\right]\right\}=\operatorname{sgn}\left\{k_{\delta}\right\} .
$$

As a consequence, as $\boldsymbol{k}$ varies in any direction from the left-side to the right-side of $\mathcal{T}_{\ell}, \forall \ell \in \mathbb{N}$ crossing through a point $\hat{\boldsymbol{k}}:=\left[\hat{k_{p}}, \hat{k_{p}}\right]$, a pair of roots of $(5)$ crosses from the LHP to the RHP of the complex plane if $\hat{k}_{\delta}>0$ for $\ell$ even or $\hat{k}_{\delta}<0$ for $\ell$ odd. Furthermore, the crossing is from the RHP to the LHP if these conditions are reversed.

\subsection{Stability index determination}

Based on the crossing directions obtained in the previous subsection, we present a conceptual example explaining these results and a novel algorithm aiming at finding the invariant number of roots $\eta$ (stability index) of a given region of the parameter space.

\subsubsection{Conceptual example}

As stated in Propositions 7-8, we refer to a positive crossing of $\boldsymbol{k}$ over $\mathcal{T}_{i}$, if the direction of $\boldsymbol{k}$ coincides with the positive direction. Otherwise, we say that we have a negative crossing of $\boldsymbol{k}$. Moreover, we define the numbers $\eta, \eta_{0} \in \mathbb{N}_{+} \cup\{0\}$, as the number of roots in the RHP, of the closed-loop system and of the open-loop system, respectively.

Example 1 (Conceptual example): Let $\tau \in \mathbb{R}_{+}$be a fixed value, and consider the particular case where $Q(j \omega) \neq 0$ for all $\omega \in \Omega \cup\{0\}$. Fig. 2 shows a posible scenario for the problem of stabilization of the closed-loop system in the $k_{p}-k_{\delta}$ parameters plane, where, as mentioned above, the arrows of the stability crossing curves $\mathcal{T}_{i}$ indicate the positive direction of $\mathcal{T}_{i}$.

In order to analyze the stability of each region in the $k_{p}-k_{\delta}$ parameters plane, we compute first the number $\eta$ of each region by analyzing the crossing directions as we vary the controller gains $\boldsymbol{k}$ continuously from $A$ to $F$, as shown in Fig. 2. From (5) it can be seen that at the origin of the $k_{p}-k_{\delta}$ parameters plane, the roots of this function are the same as the roots of the open-loop system, that is, the roots of $Q(s)$. Therefore, $\eta=\eta_{0}$ for $\boldsymbol{k}=[0,0]^{T}$, which corresponds to the point $A$ as depicted in Fig. 2.

The rest of the analysis is summarized in Table 1, where the type of crossing through $\mathcal{T}_{i}$ is indicated according to the positive direction of $\mathcal{T}_{i}$ for $i \neq 0$, and to the point $\boldsymbol{k}_{0}$ of $\mathcal{T}_{0}$ for $i=0$ (see, Proposition 8). Furthermore, $\eta_{-}$and $\eta_{+}$denote the numbers of roots in the RHP of the complex plane in the previous and future region as $\boldsymbol{k}$ crosses $\mathcal{T}_{i}$. Observe also from Fig. 2, that the only

Table 1. Results of the stability analysis: a conceptual example.

\begin{tabular}{ccccccc}
\hline Point & $\begin{array}{c}\text { Type of crossing } \\
\text { through } \mathcal{T}_{i}\end{array}$ & $i$ & $\begin{array}{c}\text { Position } \\
\text { related to } \mathbf{k}_{\mathbf{0}}\end{array}$ & $k$-direction & $\eta_{-}$ & $\eta_{+}$ \\
\hline$B$ & left to right & even & & + & $\eta_{0}$ & $\eta_{0}+2$ \\
$C$ & left to right & 0 & to the right & - & $\eta_{0}+2$ & $\eta_{0}+1$ \\
$D$ & right to left & even & & - & $\eta_{0}+1$ & $\eta_{0}-1$ \\
$E$ & right to left & odd & & + & $\eta_{0}-1$ & $\eta_{0}+1$ \\
$F$ & right to left & 0 & to the left & - & $\eta_{0}+1$ & $\eta_{0}$ \\
\hline
\end{tabular}

plausible scenario corresponds to the case when $\eta_{0} \geq 1$, since $\eta$ cannot be a negative number. Moreover, the only scenario in which we could have a stability region in the section of the $k_{p}-k_{\delta}$ parameters plane, is the one in which $\eta_{0}=1$. 


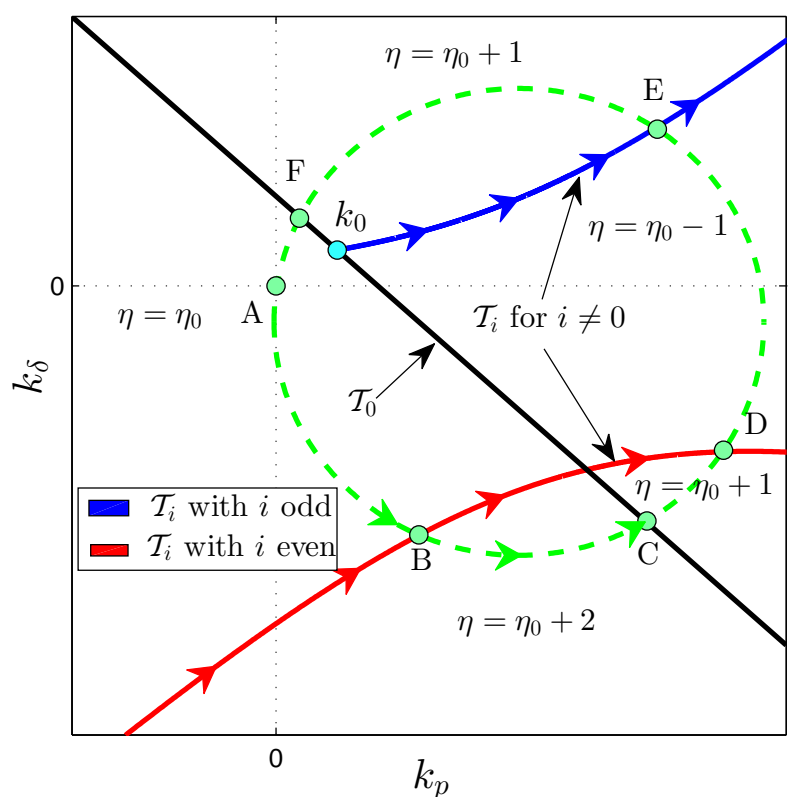

Figure 2. Conceptual example of a stability analysis using stability crossing curves.

\subsubsection{Stability index determination algorithm}

Let $\boldsymbol{k}^{*}:=\left[k_{p}^{*}, k_{\delta}^{*}\right]^{T}$ denote a point on the $k_{p^{-}} k_{\delta}$ parameters plane such that $\boldsymbol{k}^{*} \notin \mathcal{T}_{i}$ for $i \in \mathbb{N} \cup\{0\}$. We propose a linear path for $\boldsymbol{k}$ from the origin (at which $\eta=\eta_{0}$ ) to $\boldsymbol{k}^{*}$. The set $\Omega_{s}$ denote all $\omega \in \Omega$ for which $\boldsymbol{k}(\omega)$ intersects the vector $\boldsymbol{k}^{*}$. This set corresponds to all $\omega \in \Omega$ such that the following equation holds:

$$
k_{p}^{*} k_{\delta}(\omega)-k_{\delta}^{*} k_{p}(\omega)=0,
$$

and satisfies at least one of the following conditions:

$$
0<\frac{k_{p}(\omega)}{k_{p}^{*}}<1, \quad 0<\frac{k_{\delta}(\omega)}{k_{\delta}^{*}}<1
$$

Observe that there can only be one intersection between $\mathbf{k}^{*}$ and $\mathcal{T}_{0}$. This intersection exists if and only if (15) holds for $\boldsymbol{k}=\alpha \boldsymbol{k}^{*}$ where $\alpha \in(0,1)$. This leads to the definition of the indicative function $\mathcal{I}_{\alpha}$ as follows:

$$
\mathcal{I}_{\alpha}:=\left\{\begin{array}{ccc}
1 & \text { if } & \alpha \in(0,1), \\
0 & \text { if } & \alpha \notin(0,1),
\end{array}\right.
$$

where $\alpha$ is computed as:

$$
\alpha=-\frac{q_{0}}{p_{0}} \frac{1}{k_{p}^{*}+k_{\delta}^{*}}
$$

The indicative function $\mathcal{I}_{\alpha}$ establishes the existence of an intersection between $\boldsymbol{k}^{*}$ and $\mathcal{T}_{0}$ if and only if $\mathcal{I}_{\alpha}=1$. Note that the stability crossing curve $\mathcal{T}_{i}$ for $i \in \mathbb{N} \cup\{0\}$ may cross at the origin of the parameter space. That situation is related to the possible roots on the imaginary axis of the open-loop characteristic equation. Such a case must be treated separately, and for that reason we 
define the set $\Omega_{t}$ as the set of all $\omega \in \mathbb{R}_{+} \cup\{0\}$ where $Q(j \omega)=0$. Let $\mathcal{R}^{*} \subset \mathbb{R}^{2}$ denote the region where $\boldsymbol{k}^{*}$ is located. Finally, using Remark 5 , we construct the functions $\nabla$ and $\nabla_{0}$ defined as:

$$
\nabla_{0}\left(\boldsymbol{k}^{*}\right):=\operatorname{sgn}\left\{k_{p_{0}} k_{\delta}^{*}-k_{p}^{*} k_{\delta_{0}}\right\}, \quad \nabla\left(\boldsymbol{k}^{*}, \omega\right):=(-1)^{\left\lceil\frac{\tau}{\pi} \omega\right\rceil} \operatorname{sgn}\left\{k_{p}^{*} k_{\delta}^{\prime}(\omega)-k_{p}^{\prime}(\omega) k_{\delta}^{*}\right\}
$$

where $k_{p}^{\prime}(\omega)$ and $k_{\delta}^{\prime}(\omega)$ stand for the derivatives of $k_{p}(\omega)$ and $k_{\delta}(\omega)$ with respect to $\omega$, respectively. We have the following result:

Proposition 10: Let $G$ be a non even function with $\operatorname{deg} Q>\operatorname{deg} P, \tau \in \mathbb{R}_{+}$be a fixed value and let $\boldsymbol{k}^{*}:=\left[k_{p}^{*}, k_{\delta}^{*}\right]^{T} \in \mathcal{R}^{*} \subset \mathbb{R}^{2}$ such that $\boldsymbol{k}^{*} \notin \mathcal{T}_{i}, \forall i \in \mathbb{N} \cup\{0\}$. For $f(j \omega):=G^{-1}(j \omega)$ assume that the following condition holds:

$$
-\Im\{f(j \omega)\}+\frac{1}{\tau} \Re\left\{f^{\prime}(j \omega)\right\} \neq 0, \quad \forall \omega \in \Omega_{s} .
$$

If $\Omega_{t}=\emptyset$, then, $\forall \boldsymbol{k} \in \mathcal{R}^{*}$ the number of roots $\eta$ on the RHP of the complex plane of (5) can be computed by:

$$
\eta=\eta_{0}+\mathcal{I}_{\alpha} \nabla_{0}\left(\boldsymbol{k}^{*}\right)+2 \sum_{\omega \in \Omega_{s}} \nabla\left(\boldsymbol{k}^{*}, \omega\right)
$$

Furthermore, if $\Omega_{t} \neq \emptyset$, then $\forall \boldsymbol{k} \in \mathcal{R}^{*}$ the number of roots $\tilde{\eta}$ on the RHP of the complex plane of (5) is given by:

$$
\tilde{\eta}=\eta+\sum_{\omega \in \Omega_{t}}\left\{\frac{(1-\operatorname{sgn}(\omega))\left(\nabla_{0}\left(\boldsymbol{k}^{*}\right)+1\right)+2 \operatorname{sgn}(\omega)\left(\nabla\left(\boldsymbol{k}^{*}, \omega\right)+1\right)}{2}\right\}
$$

Proof. Observe first that if condition (66) holds, (5) does not have multiple roots, hence Propositions 7 and 8 are valid. Consider now the fixed values $\tau, \boldsymbol{k}^{*}, \eta_{0}$ and $\alpha$, as well as the sets $\Omega_{s}$ and $\Omega_{t}$, and the functions $\mathcal{I}_{\alpha}, \nabla_{0}\left(\boldsymbol{k}^{*}\right)$ and $\nabla\left(\boldsymbol{k}^{*}, \omega\right)$, as defined above.

It is clear that in case where $\Omega_{t}=\emptyset$, this implies that $\tilde{\eta}=\eta$. Thus, in the remaining part of the proof, we consider only the computation of $\tilde{\eta}$. In order to determine $\tilde{\eta}$ for $\mathcal{R}^{*}$, we need to observe the behavior of the roots of the characteristic equation (5) as $\boldsymbol{k}$ varies from the origin to $\boldsymbol{k}^{*}$. Evidently, $\tilde{\eta}=\eta_{0}$ for $\boldsymbol{k}=\mathbf{0}$; then starting from such a point, we need to analyze the behavior of the roots as $\boldsymbol{k}$ varies along the vector $\boldsymbol{k}^{*}$, where four possible scenarios can be identified:

(i) The vector $\boldsymbol{k}^{*}$ crosses through the stability crossing curve $\mathcal{T}_{0}$.

(ii) The vector $\boldsymbol{k}^{*}$ crosses through at least one stability crossing curve $\mathcal{T}_{i}$ for $i \in \mathbb{N}$.

(iii) $\mathcal{T}_{0}$ crosses through the origin of the $k_{p}-k_{\delta}$ parameters plane.

(iv) At least one $\mathcal{T}_{i}$ for $i \in \mathbb{N}$ crosses through the origin of the $k_{p}-k_{\delta}$ parameters plane.

The existence of these scenarios is described by the function $\mathcal{I}_{\alpha}$ as well as by means of the sets $\Omega_{s}$ and $\Omega_{t}$. For the cases (i) and (iii), a crossing of one root through the origin of the complex plane occurs, and according to Proposition 8, this root is crossing to the RHP of the complex plane if the vector $\boldsymbol{k}^{*}$ is located at the left of the vector related to $\boldsymbol{k}_{0}$, otherwise, the crossing is to the LHP. In any cases, such a behaviour is taking into account by $\nabla_{0}\left(\boldsymbol{k}^{*}\right)$. Now, for the cases (ii) and (iv) the crossings are through the imaginary axis of the complex plane. Then, according to Proposition 7 , the crossing are to the RHP of the complex plane if the vector $\boldsymbol{k}^{*}$ is located to the right of $\mathcal{T}_{i}$ with respect to the positive direction of $\mathcal{T}_{i}$ for $i$ even (or to the left for $i$ odd), otherwise, the crossing is to the LHP. This behavior is represented by the function $\nabla\left(\boldsymbol{k}^{*}, \omega\right)$.

The proof ends by noting that the total counting of roots, considering all possible scenarios (i)-(iv), are summarized by means of the expression (67). 
Remark 6: It is noteworthy that although Proposition 10 is restricted to non even open-loop systems and non neutral closed-loop systems, it can be easily extended using the ideas introduced in Section 4 and Proposition 4, respectively.

\subsubsection{First unstable regions}

Recall the ideas stated in the conceptual example shown in section 4.2.1 and Propositions 5 and 8. Particulary, from Section 4.2.1, we highlight the behavior of the roots of the characteristic equation as a real crossing is achieved in the same manner as is shown in points $C$ and $F$ from Fig. 2.

Notice that the line (3) partitions the $k_{p}-k_{\delta}$ parameters space in two unbounded regions. For brevity, we denote the regions above and under the line (3) as $R_{+}$and $R_{-}$, respectively. Given this notation we shown the following result:

Proposition 11: Let $P(s) \neq 0$ for all $s>0, R_{+}$and $R_{+}$be unbounded regions in the $k_{p}-\delta$ parameters space as already defined and $\mathcal{T}_{u}$ be the curve explicitly defined in Proposition 5. Then, the following is true:

$$
\begin{aligned}
& \text { If } \mathcal{T}_{u} \subseteq R_{+}, \quad \text { then, } R_{-} \text {is a unstable region, } \\
& \text { If } \mathcal{T}_{u} \subseteq R_{-}, \quad \text { then, } R_{+} \text {is a unstable region. }
\end{aligned}
$$

Proof. First of all, let us remind some basic behavior of the roots of a quasi-polynomial with real coefficients. Fig. 3 shows a possible movement of two roots which starts as real and ends in a complex conjugate form. Basically, as shown in the point $H$ of Fig. 3, we are interested in the fact that a real root can become a complex root only by intersecting with a secondary real root to finally move out from the real axis.

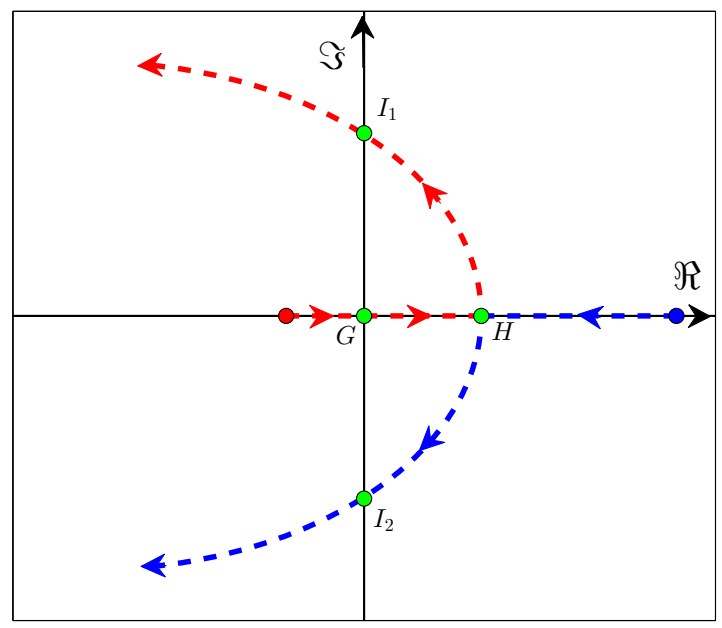

Figure 3. Real Root Crossing Particular Behavior.

The point $H$ corresponds to setting the controller gains in a point on the curve $\mathcal{T}_{u}$ defined in Proposition 5. Furthermore, the curve $\mathcal{T}_{u}$ is a continuous curve and is contained entirely in the region $R_{+}$or in the region $R_{-}$if $P(s) \neq 0$ for all $s>0$. The proof of this is based in the fact that the only point of the line (3) in which the characteristic equation has a multiple real root is $k_{0}$ 
and that $\mathcal{T}_{u}$ is a continuos curve if $P(s) \neq 0$ for all $s>0$.

Then, consider a parametrical variation of the controller gains $k_{p}$ and $k_{\delta}$ such that $\boldsymbol{k}$ starts in $R_{+}$and then traverses from right to left through the line (3) at any point at the right side of $k_{0}$, moving to $R_{-}$. As stated in Proposition 8, this implies that the characteristic equation of the closed loop system gains a real root with positive real part (as shown conceptually in the point $G$ of Fig. 3). Given the fact that if $\mathcal{T}_{u}$ is located in $R_{+}$, for every parametrical variations of the controller gains inside the region $R_{-}$, this real root does not crosses to the LHP of the complex plane in the same manner that in the points $I_{1}$ and $I_{2}$ from Fig. 3 . Therefore, $R_{-}$is an unstable region.

The proof ends by developing a similar analysis considering a parametrical variation of $\boldsymbol{k}$ starting in $R_{-}$and then traversing from left to right through the line (3) at any point at the left side of $k_{0}$, moving to $R_{+}$.

\section{Performance and fragility of $P-\delta$ controllers}

As mentioned in the introduction, we discuss briefly two important issues in the controllers design. The first one concerns the exponential decay of the system response which gives a measure of the performance of the closed-loop system. The second one is the controllers fragility analysis which gives a measure of the robustness of the closed-loop system against parametrical uncertainties in the control gains.

\section{$5.1 \quad \sigma-S t a b i l i t y$}

Let $\sigma \in \mathbb{R}_{-}$, the $\sigma$-stability problem can be described as the task of determining the controller gains $\boldsymbol{k}$ such that the real part of the rightmost roots of the characteristic equation (5) is smaller than $|\sigma|$.

Let $\mathcal{T}_{\sigma}$ denote the set of all $\boldsymbol{k}$ such that (5) has at least one root on the vertical line of the complex plane defined as $\boldsymbol{L}_{\sigma}:=\sigma+j \omega$ for all $\omega \in \mathbb{R}$. This vertical line is defined as the $\sigma$-axis. In order to introduce similar results to those presented in Section 3 , let us consider the set $\Omega_{\sigma}$, defined as

$$
\Omega_{\sigma}:=\left\{\omega \in \mathbb{R} \mid \omega \neq \frac{\pi}{\tau} i \text { for } i \in \mathbb{N} \cup\{0\} \text { and } P(\sigma+j \omega) \neq 0\right\} .
$$

We have the following:

Corollary 1: Let $\omega \in \Omega_{\sigma}$, and let $\tau \in \mathbb{R}_{+}, \sigma \in \mathbb{R}_{-}$be fixed values. Then, the set $\mathcal{T}_{\sigma}$ can be computed as:

$$
\mathcal{T}_{\sigma}=\mathcal{T}_{\bar{\sigma}} \cup \mathcal{T}_{\sigma, 0},
$$

with

$$
\mathcal{T}_{\sigma, 0}=\left\{\boldsymbol{k} \in \mathbb{R}^{2} \mid k_{\delta}=-e^{\sigma \tau}\left\{k_{p}+\frac{Q(\sigma)}{P(\sigma)}\right\}\right\} \text { and } \mathcal{T}_{\bar{\sigma}}=\left\{\boldsymbol{k} \in \mathbb{R}^{2} \mid \boldsymbol{k}=\boldsymbol{k}_{\sigma}(\omega)\right\}
$$


where $\boldsymbol{k}_{\sigma}(\omega):=\left[k_{p_{\sigma}}(\omega), k_{\delta_{\sigma}}(\omega)\right]^{T}$, and $k_{p_{\sigma}}$ and $k_{p_{\delta}}$ are defined by:

$$
\begin{aligned}
& k_{p_{\sigma}}(\omega)=-\Re\left[\frac{Q(\sigma+j \omega)}{P(\sigma+j \omega)}\right]-\cot (\tau \omega) \Im\left[\frac{Q(\sigma+j \omega)}{P(\sigma+j \omega)}\right], \\
& k_{\delta_{\sigma}}(\omega)=e^{\tau \sigma} \csc (\tau \omega) \Im\left[\frac{Q(\sigma+j \omega)}{P(\sigma+j \omega)}\right] .
\end{aligned}
$$

Proof. By setting $s=\sigma+j \omega$, the proof follows the steps of the proofs of Propositions 3 and 7 . For the sake of brevity, it is omitted.

Remark 7: The regions found to be stable are delimited by the stability crossing curves described in the previous subsections. These boundaries are the points at which the rightmost roots are located precisely on the imaginary axis. Thus, according to Remark 1, the curves change continuously as $\sigma$ is varied continuously, and they tend to the stability crossing curves as $\sigma \rightarrow 0$. It is worth noting that the results presented in the previous section for $\sigma=0$ can be easily extended to the case $\sigma \neq 0$.

\section{2 $\quad$ Fragility}

Consider now the fragility problem, which consists in computing the maximum controller parameters deviation $d$ of a given stabilizing controller $\overline{\boldsymbol{k}}:=\left(\bar{k}_{p}, \bar{k}_{\delta}\right)^{T}$, such that the closed-loop system remains stable, as long as the controller parameters $\boldsymbol{k}$ satisfy the inequality:

$$
\sqrt{\left(k_{p}-\bar{k}_{p}\right)^{2}+\left(k_{\delta}-\bar{k}_{\delta}\right)^{2}}<d .
$$

In order to address this problem, let us introduce the following notations. First, let $\boldsymbol{k}(\omega)=$ $\left[k_{p}(\omega), k_{\delta}(\omega)\right]^{T}$ as given in Proposition 1 and let us introduce the function $\xi: \mathbb{R}_{+} \rightarrow \mathbb{R}_{+}$defined as

$$
\xi(\omega):=\sqrt{\left(k_{p}(\omega)-\bar{k}_{p}\right)^{2}+\left(k_{\delta}(\omega)-\bar{k}_{\delta}\right)^{2}}
$$

We have the following:

Proposition 12: Let $\overline{\boldsymbol{k}}$ be a stabilizing controller. Then, the maximum parameter deviation $d$ of $\overrightarrow{\boldsymbol{k}}$, such that the closed-loop system remains stable, are given by:

$$
d:=\min \left\{\tilde{d}, d_{o}, d_{n}, d_{\ell}\right\}
$$

with $\tilde{d}$ given by:

$$
\tilde{d}:=\min _{\omega \in \Omega_{f}}\{\xi(\omega)\}
$$

where $\Omega_{f}$ denote the set of all roots of $f(\omega)$ defined as:

$$
f(\omega):=\left\langle\boldsymbol{k}(\omega)-\overline{\boldsymbol{k}}, \boldsymbol{k}^{\prime}(\omega)\right\rangle,
$$


and where $d_{o}, d_{n}$ and $d_{\ell}$ can be computed by:

$$
\begin{aligned}
& d_{o}=\frac{1}{\sqrt{2}}\left|\bar{k}_{p}+\bar{k}_{\delta}+\frac{q_{0}}{p_{0}}\right|, \\
& d_{n}=\left\{\begin{array}{ll}
\min \left\{\frac{1}{\sqrt{2}}\left|\bar{k}_{\delta} \pm\left\{\bar{k}_{p}+\frac{q_{n}}{p_{n}}\right\}\right|\right\} & \text { if } \operatorname{deg} Q=\operatorname{deg} P \\
\infty & \text { if } \operatorname{deg} Q>\operatorname{deg} P
\end{array},\right. \\
& d_{\ell}= \begin{cases}\min _{\ell \in \mathbb{N}}\left\{\frac{1}{\sqrt{2}}\left|\bar{k}_{\delta}(-1)^{\ell}\left\{\bar{k}_{p}+\frac{Q\left(j \omega_{\ell}\right)}{Q\left(j \omega_{\ell}\right)}\right\}\right|\right\} & \text { if } G \text { is an even function } \\
\infty & \text { if } G \text { is not even }\end{cases}
\end{aligned}
$$

Proof. By assumption $\overline{\boldsymbol{k}}$ is located inside some stability region delimited by some appropriate stability crossing curves, thus, the closed-loop system is unstable if the controller $\overline{\boldsymbol{k}}$ has a parameter deviation such that it crosses for at least one of its boundaries. Therefore, the objective is to compute the minimal distances between $\overline{\boldsymbol{k}}$ and the different boundaries of the stability region. In order to compute the minimal distance between a point $\overline{\boldsymbol{k}}$ and the stability crossing curves with $\omega \neq 0$, we need to identify the points $\boldsymbol{k}(\omega)$ at which the tangent vectors to the curve are orthogonal to $\boldsymbol{k}(\omega)-\overline{\boldsymbol{k}}$. In other words, to find points in which $\omega$ is a root of equation (79). Therefore, the minimum distance $\tilde{d}$ to a stability crossing curve with $\omega \neq 0$ is given by (78). Observe also that the boundaries of the stability crossing curve related to $\omega=0$ are described by the line (15). Thus, the minimum distance to this line can be computed as follows:

Substituting (15) in (76) leads to

$$
\xi(0)=\sqrt{\left(k_{p}-\bar{k}_{p}\right)^{2}+\left(k_{p}+\frac{q_{0}}{p_{0}}+\bar{k}_{\delta}\right)^{2}},
$$

the gain $k_{p}$ at which $\xi(0)$ attains its minimum, is given by the solution of the following equation:

$$
\frac{d \xi^{2}(0)}{d k_{p}}=4 k_{p}+2\left(\bar{k}_{p}-\bar{k}_{\delta}+\frac{q_{0}}{p_{0}}\right)=0 .
$$

Then, this value is defined as $d_{o}$ and can be obtained by substituting the solution of (84) in (83). A similar process can be further derived for the stability crossing curves of neutral or even systems, which results in the distances $d_{n}$ and $d_{\ell}$. From the above analysis, it is clear that the minimal distance $d$ is given by (77).

\section{Numerical examples}

In order to illustrate the effectiveness of the proposed results, we consider several numerical examples.

Example 2 (Marginally stable fourth-order system): Consider a system with the following transfer function:

$$
G(s)=\frac{0.038}{s^{4}+0.1276 s^{3}+9.3364 s^{2}+1.1484 s+3.0276}
$$

subject to the $P-\delta$ controller $C(s)=k_{p}+k_{\delta} e^{-\tau s}$. The open-loop poles of the system are located at $s=-0.0638 \pm 0.5765 j$ and $s= \pm 3 j$, which means that the system is marginally stable. In order 
to illustrate the proposed results, let consider a fixed delay $\tau=5$. According to Proposition 1, we have:

$$
\begin{aligned}
& k_{p}(\omega)=\left(3.35789 \omega^{2}-30.2211\right) \omega \cot (\tau \omega)-26.3158 \omega^{4}+245.695 \omega^{2}-79.6737 \\
& k_{\delta}(\omega)=\omega\left(30.2211-3.35789 \omega^{2}\right) \csc (\tau \omega) .
\end{aligned}
$$

Considering (86) along with Propositions 1 and 3, we obtain the stability crossing curves depicted in Fig.4 (left). Now, as can be seen in Fig. 4 (right) with the purpose to discriminate unstable regions avoiding unnecessary computations, we plot the $\mathcal{T}_{u}$-curve by applying Proposition 5. Next, in order to find the stability regions, we apply Proposition 10, leading to the result illustrated in Fig. 4 (right). To illustrate how Proposition 10 is applied, let us consider three points $\boldsymbol{k}_{1}^{*}$, $\boldsymbol{k}_{2}^{*}$ and $\boldsymbol{k}_{3}^{*}$ on the parameter space. The results are summarized in table 2.

Table 2. Results of the stability analysis algorithm-Marginally stable fourth-order system.

\begin{tabular}{ccccccccc}
\hline Point & $k_{p}$ & $k_{\delta}$ & $\mathcal{I}_{\alpha}$ & $\Omega_{t}$ & $\Omega_{s}$ & $\nabla, \omega \in \Omega_{t}, \Omega_{s}$ & $\eta_{0}$ & $\eta$ \\
\hline $\boldsymbol{k}_{1}^{*}$ & 400 & 200 & 0 & $\{1.3278,2.5451\}$ & $\{3\}$ & $\{1,1\},\{-1\}$ & & 4 \\
$\boldsymbol{k}_{2}^{*}$ & 350 & -25 & 0 & $\{\emptyset\}$ & $\{3\}$ & $\{0\},\{1\}$ & 0 & 2 \\
$\boldsymbol{k}_{3}^{*}$ & 500 & -200 & 0 & $\{0.8395,1.2150,1.9358\}$ & $\{3\}$ & $\{1,-1,1\},\{1\}$ & \\
\hline
\end{tabular}
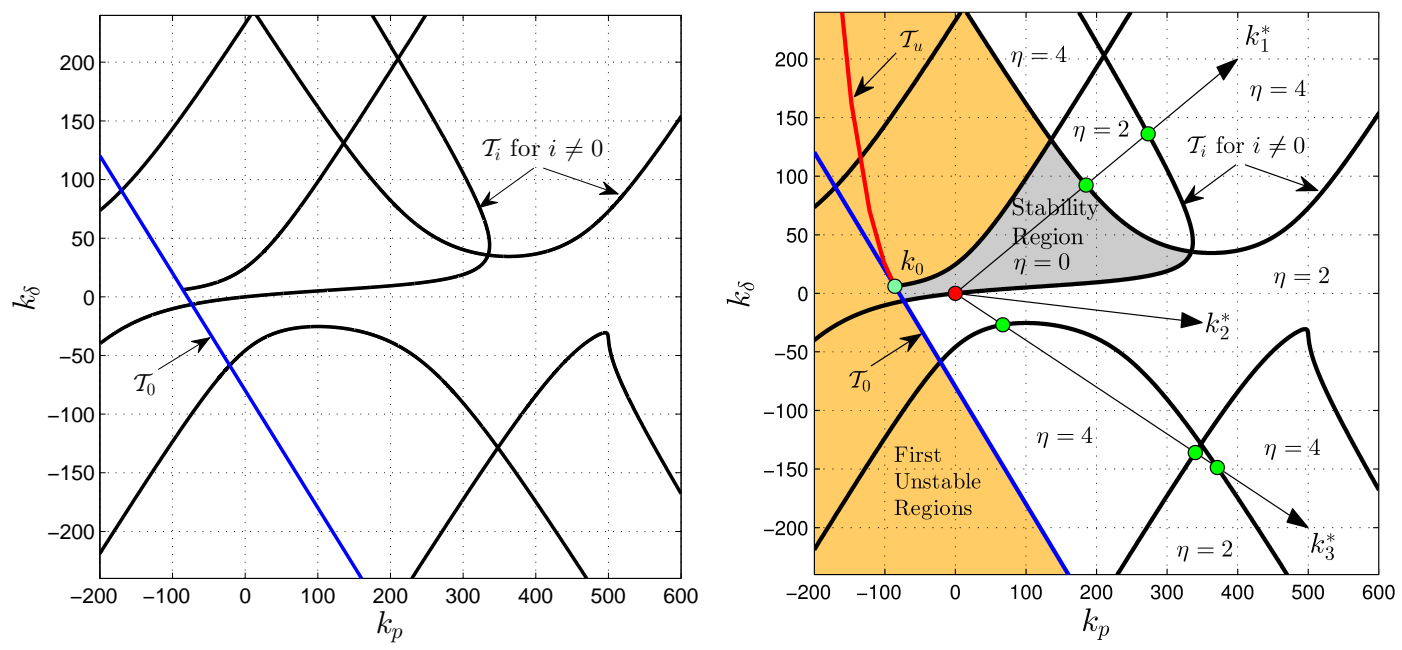

Figure 4. Marginally stable fourth-order system (88). (Left) Stability crossing curves. (Right) Stability Regions.

Now, in order to show the applicability of Proposition 12, consider the controllers $\widetilde{\boldsymbol{k}}_{1}, \widetilde{\boldsymbol{k}}_{2}$ and $\widetilde{\boldsymbol{k}}_{3}$ located inside the stable region, see, for instance, Fig. 5 (left). Next, after applying Proposition 12, we get the results summarized in table 3 , where according to this table, the controller $\widetilde{\boldsymbol{k}}_{2}$ is the less fragile.

Table 3. Fragility analysis - Marginally stable fourthorder system.

\begin{tabular}{cccccc}
\hline Point & $k_{p}$ & $k_{\delta}$ & $\tilde{d}$ & $d_{0}$ & $d$ \\
\hline$\tilde{\mathbf{k}}_{1}$ & -72 & 1 & 6.2996 & 6.1332 & 6.1332 \\
$\tilde{\mathbf{k}}_{2}$ & 135 & 60 & 51.6999 & 194.2236 & 51.6999 \\
$\tilde{\mathbf{k}}_{3}$ & 270 & 30 & 15.3476 & 268.4698 & 15.3476 \\
\hline
\end{tabular}



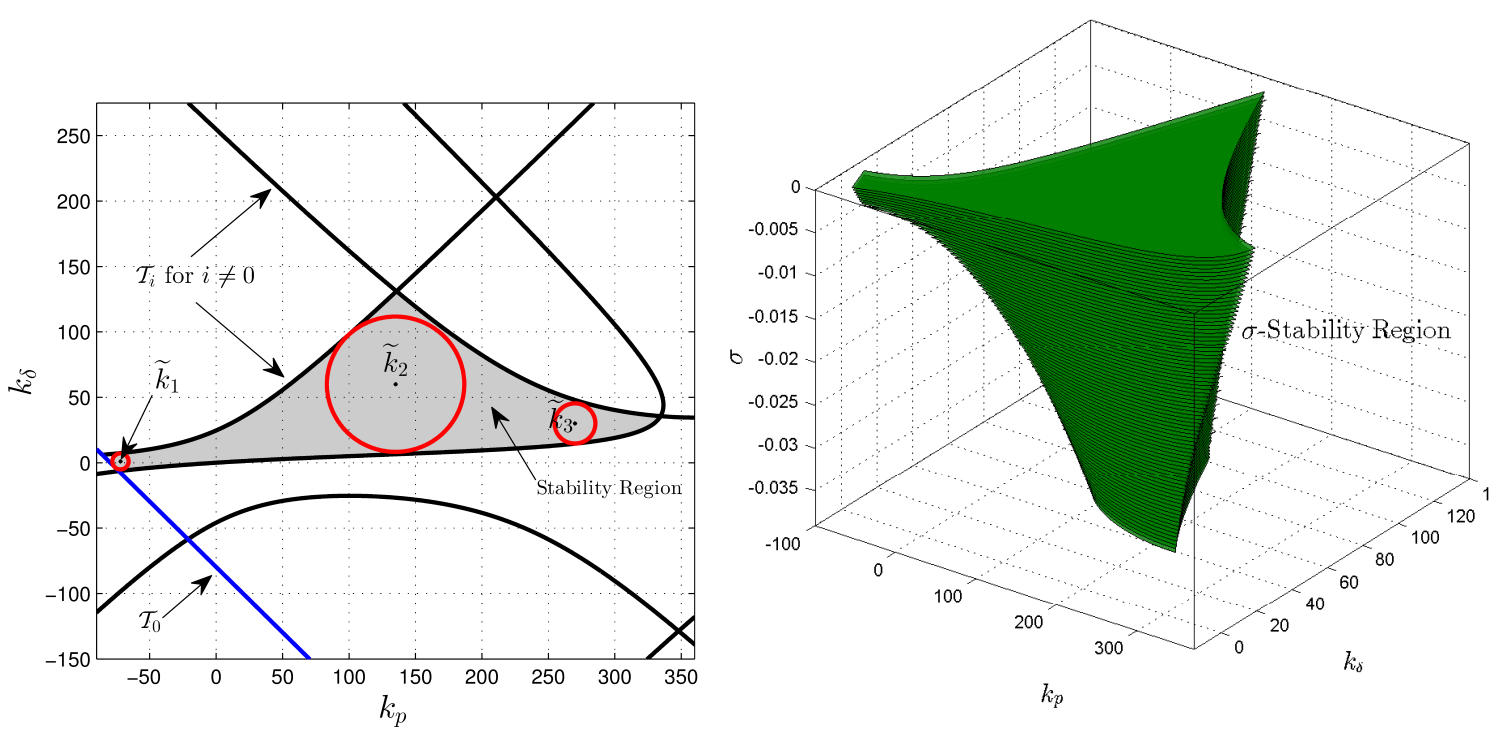

Figure 5. Marginally stable fourth-order system example. (Left) Fragility analysis. (Right) $\sigma$-stability region behavior through the $\sigma$-axis.

Finally we consider the $\sigma$ stability problem, where by Corollary 1, we obtain the result depicted in Fig.5 (right). Moreover, such a figure depicts the behavior of the rightmost roots of the closed-loop system, as $\sigma$ varies from 0 to -0.035 . We can conclude that any controller $\boldsymbol{k}$, in each level surface with a particular $\sigma$ value implies that the closed-loop system has all of its roots located at least to a distance $|\sigma|$ from the imaginary axis.

Example 3 (Neutral-type system): Consider a system with the following transfer function:

$$
G(s)=\frac{s^{6}-5 s^{5}+20 s^{4}-10 s^{3}+5 s+1}{s^{6}+s^{4}+4 s^{3}+7 s^{2}+9 s+1} .
$$

We propose a fixed delay $\tau=0.5$ for the stabilization of the closed-loop system using a $P-\delta$ controller. It is clear that this system is of neutral type since $\operatorname{deg} Q=\operatorname{deg} P$. Therefore, in order to characterize all possible stability crossing curves, we must consider the result shown in Proposition 4 in addition to the Propositions 1 and 3. Notice that all the points of the $k_{p}-k_{\delta}$ parameters plane which do not satisfy the condition proposed in Proposition 4 implies that the closed-loop system is unstable. Fig. 3 shows the application of this result denoted as the neutral type condition.

Finally, in order to characterize each region bounded by the stability crossing curves, we use the stability analysis algorithm proposed in Proposition 10 for at least one point in each region which does not satisfy the neutral type condition. Fig. 3 illustrate the application of this proposition for three points $\boldsymbol{k}_{i}^{*}$, with $i \in\{1,2,3\}$. The results of each analysis are summarized in table 3 and illustrated in Fig 3.

Table 4. Results of the stability analysis algorithm: neutral-type system.

\begin{tabular}{cccccccccc}
\hline Point & $k_{p}$ & $k_{\delta}$ & $\mathcal{I}_{\alpha}$ & $\Omega_{t}$ & $\Omega_{s}$ & $\nabla_{0}$ & $\nabla$ & $\eta_{0}$ & $\eta$ \\
\hline$\tilde{\mathbf{k}}_{1}^{*}$ & 2 & 2 & 1 & & $\{0.9002,3.1862\}$ & -1 & $\{-1,-1\}$ & & 0 \\
$\tilde{\mathbf{k}}_{2}^{*}$ & 2 & 0.5 & 1 & $\{\emptyset\}$ & $\{0.8357\}$ & -1 & $\{-1\}$ & 5 & 2 \\
$\tilde{\mathbf{k}}_{3}^{*}$ & 1.5 & -1.5 & 0 & & $\{0.4982\}$ & & $\{-1,-1\}$ & & 3 \\
\hline
\end{tabular}




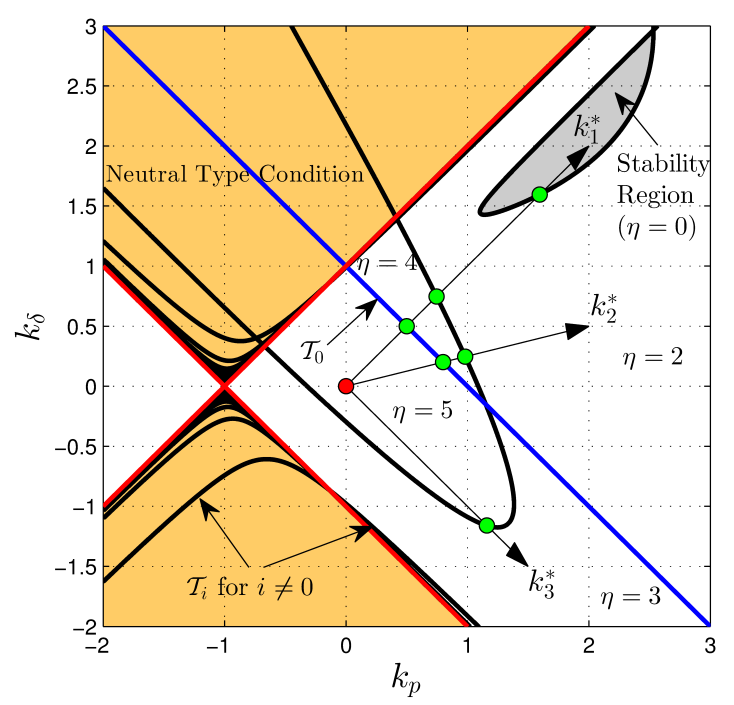

Figure 6. Algorithm example - Neutral type system.

Example 4 (Even-type system): Consider the following system (Niculescu, 2001):

$$
G(s)=\frac{1}{s^{2}+1} .
$$

This system is a even-type system, these can be easily verified by substituting $s=j \omega$ in the transfer function shown above. Therefore, it must be analyzed by using the results shown in section 5 . We propose a fixed delay $\tau=1$ for the stabilization of the closed-loop system using a P- $\delta$ controller.

The construction of the stability crossing curves $\mathcal{T}_{0}, \mathcal{T}_{p_{\ell}}$ and $\mathcal{T}_{i}$ is obtained using Propositions 3 and 2 and the crossing directions analysis is computed by the Propositions 7 and 9. Fig. 7 illustrates the behavior of the roots when $\boldsymbol{k}$ varies continuously from the point $A$ to the point $F$. Notice that the starting point $A$ corresponds to the characteristic equation of the open loop system, and consequently, at this point this equation has two roots on the imaginary axis. The results of this analysis can be found in table 5.

Table 5. Results of the stability analysis: even function example.

\begin{tabular}{cccccccc}
\hline Point & $\begin{array}{c}\text { Type of crossing } \\
\text { through } \mathcal{T}_{i}\end{array}$ & $i$ & $\ell$ & $\begin{array}{c}\text { Position } \\
\text { related to } k_{\delta}\end{array}$ & $k$-direction & $\eta_{-}$ & $\eta_{+}$ \\
\hline$A$ & left to right & odd & & & - & & 0 \\
$B$ & left to right & & odd & $<0$ & + & 0 & 2 \\
$C$ & right to left & even & & & - & 2 & 0 \\
$D$ & left to right & & even & $>0$ & + & 0 & 2 \\
$E$ & right to left & & odd & $>0$ & + & 2 & 4 \\
$F$ & right to left & & even & $>0$ & - & 4 & 2 \\
\hline
\end{tabular}

In order to study the stability of the closed-loop system along the $k_{p}-k_{\delta}$ parameters plane, this type of analysis can be easily extended as shown in Fig. 7. Furthermore, we study three particular stability regions by analyzing the explicitly the behavior of these regions as $\tau$ is varied from 1 to 3.5 as shown in Fig. 8. Bearing in mind Remark 1, all the points inside this geometric shape stabilize the closed-loop system for any fixed value $\tau$ in the interval $[1,3.5]$.

Example 5 (Unstable second-order system): In order to illustrate the case analyzed in Proposition 6 , that is, the case where exists an infinite number of multiple crossing frequencies (for different 

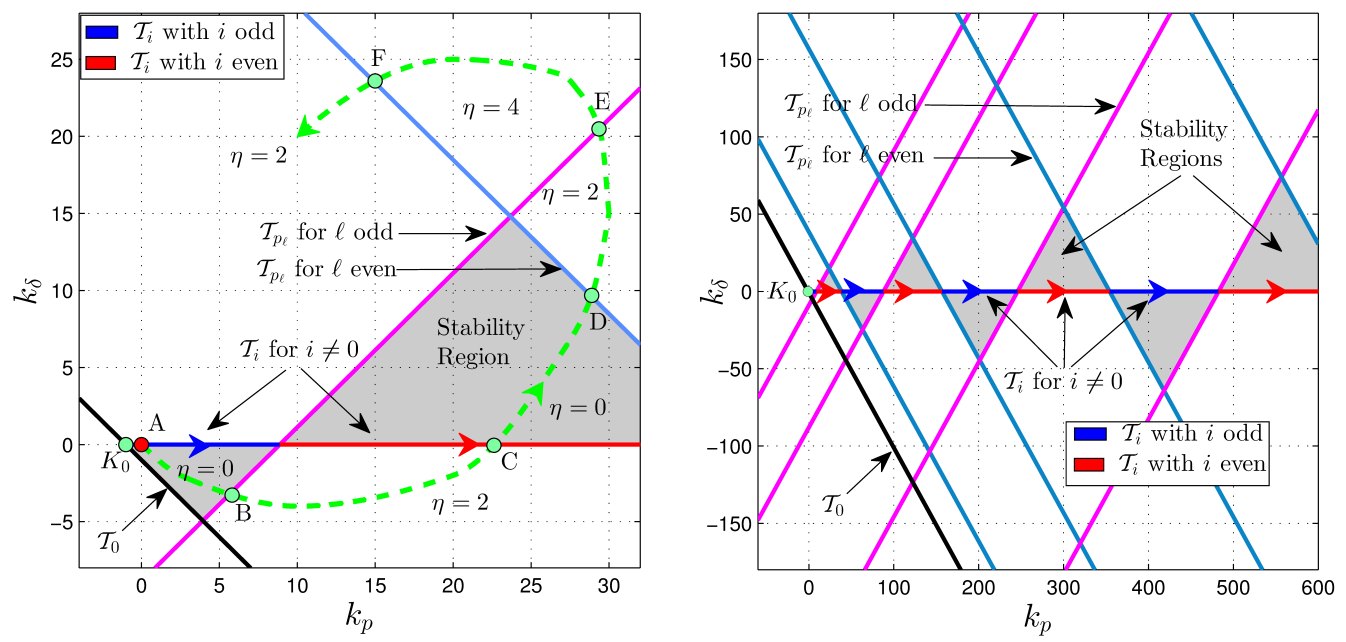

Figure 7. Stability analysis - Even function example.

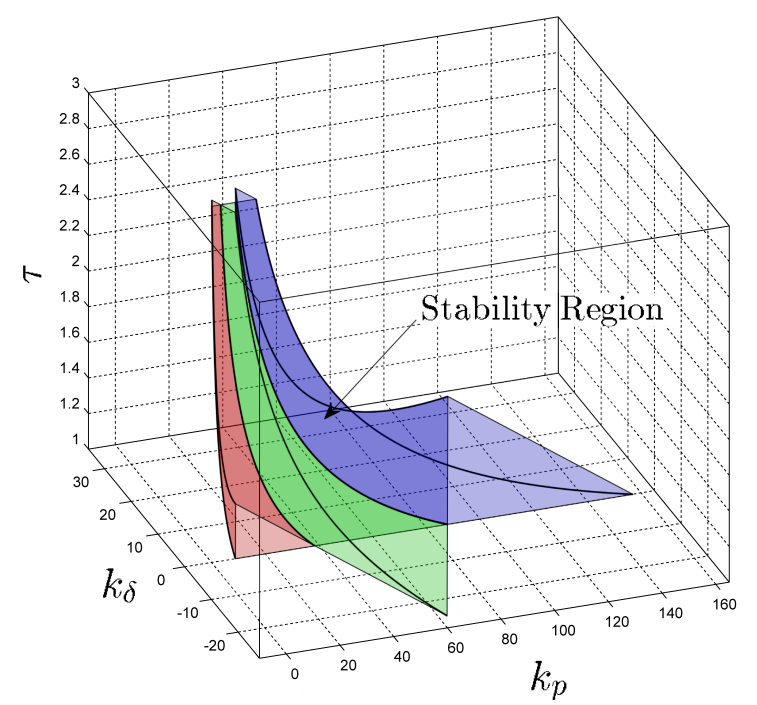

Figure 8. Stability region behavior through the $\tau$-axis - Even function example.

gain parameters), let us consider the following second-order system:

$$
G(s)=\frac{1}{-s^{2}+s+1} .
$$

Now, since condition (29) is fulfilled, i.e.,

$$
\frac{\Im\{f(j \omega)\}}{\Re\left\{f^{\prime}(j \omega)\right\}}=\frac{1}{2}>0
$$

according to Proposition 6, we have that system (89) has an infinite but countable crossing frequencies of multiplicity $m \geq 2$, if we choose $\tau=2$. Then, in order to illustrate such a phenomenon, we take the linear path $\overrightarrow{\boldsymbol{k}}$ shown in Fig. 9 (left). The rightmost root-locus is depicted in Fig. 9 (right), where we can appreciate the multiplicity of the crossing frequency. Finally, others points corresponding to a multiple crossing frequencies are indicated by the "square points". 

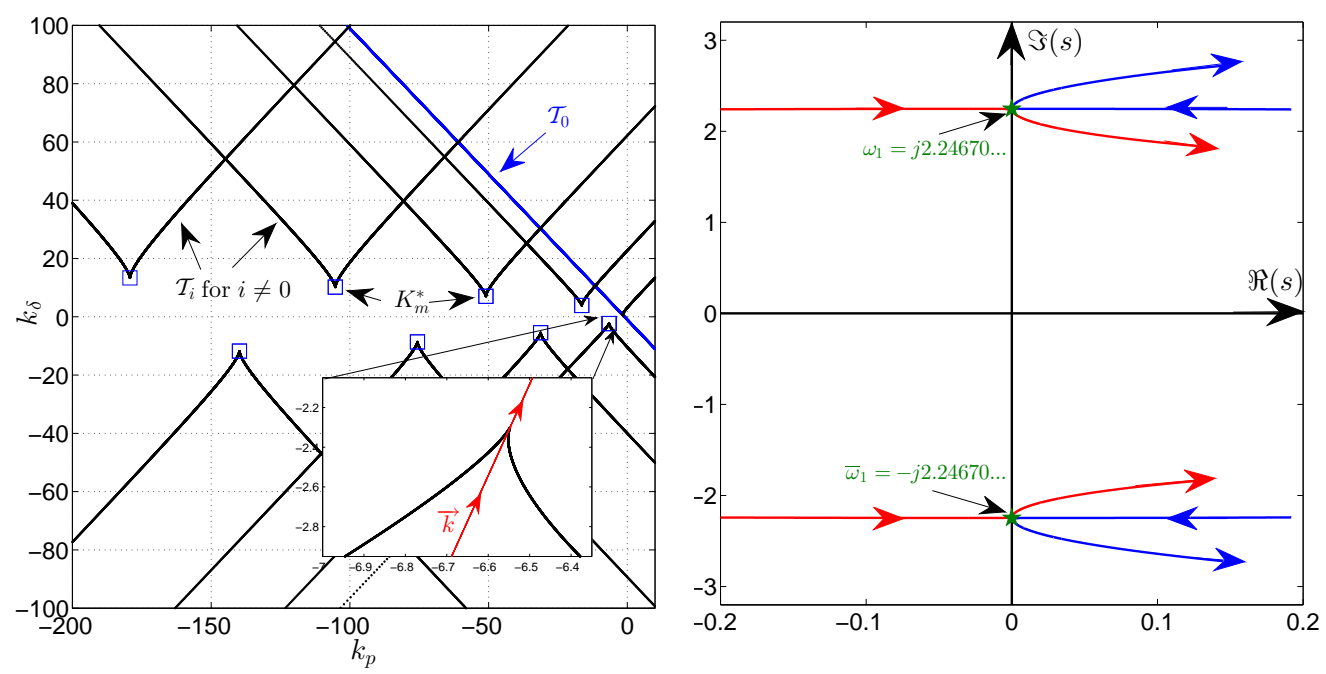

Figure 9. Marginally stable fourth-order system (88). (Left) Stability crossing curves. (Right) Stability Regions.

\subsection{Experimental results (haptic-virtual system.)}

As an experimental application of the $P-\delta$ controller, consider the haptic-virtual system developed in (Hernández-Díez et al., 2016). This experimental setup, shown in Fig. 10, consists in a haptic device working in a virtual environment with the main purpose of providing the human operator a perfect telepresence on the virtual environment and a full sense feedback of it. In order to fulfill this objective, we use a $P-\delta$ controller to achieve a kinematic correspondence between the haptic device, and a virtual device simulated on the virtual environment. The dynamics of the haptic

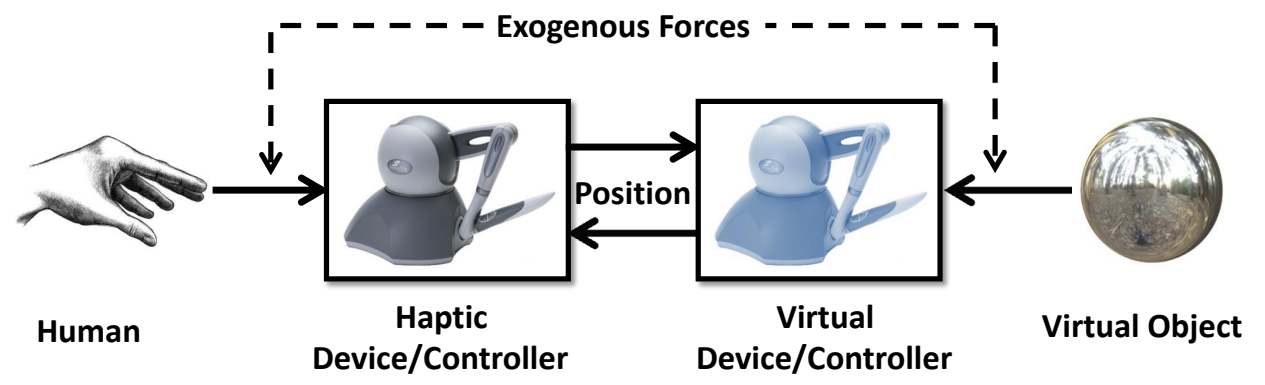

Figure 10. Bilateral control scheme.

system is described by a decoupled time-invariant linear model, with three mechanical admittances describing the dynamics of each joint, modelled as:

$$
G(s):=\frac{\Theta(s)}{\Lambda(s)}=\frac{1}{s(m s+b)}
$$

where $G(s)$ is the transfer function from each torque input $\Lambda(s)$ to its respective angular position $\Theta(s)$. According with (Hernández-Díez et al., 2016), the characteristic equation is:

$$
\Delta\left(s ; k_{p}, k_{\delta}, \tau\right):=m s^{2}+b s+2 k_{p}+2 k_{\delta} e^{-\tau s}=0 .
$$

The stability of the closed-loop system is studied using the methodology introduced in this paper. For brevity, the stability analysis is focused at the base joint, which has parameters $m=0.0131$ and 
$b=0.0941$ (these parameters are obtained using a least squares methodology, (see (Hernández-Díez et al., 2016) for more details). A fixed delay value $\tau=0.1$ is proposed for the controller design. The angular position of the haptic and virtual devices are normalized with respect to the mechanical stops of each joint and are defined as $\theta_{h}$ and $\theta_{v}$, respectively.

Fig. 11 shows the stability region of the closed loop system. In order to observe the qualitative behaviour of the system response for this particular joint, the system is tested for a fixed $k_{p}=10$ and three particular values of the delay term gain, $k_{\delta}=-1, k_{\delta}=2.6$ and $k_{\delta}=4$, corresponding to a stable point, a stable point near some stability crossing curve and an unstable point, respectively. The results are illustrated in Fig. 11, where oscillations of greater amplitude appear as $\boldsymbol{k}$ approaches the stability crossing curve.
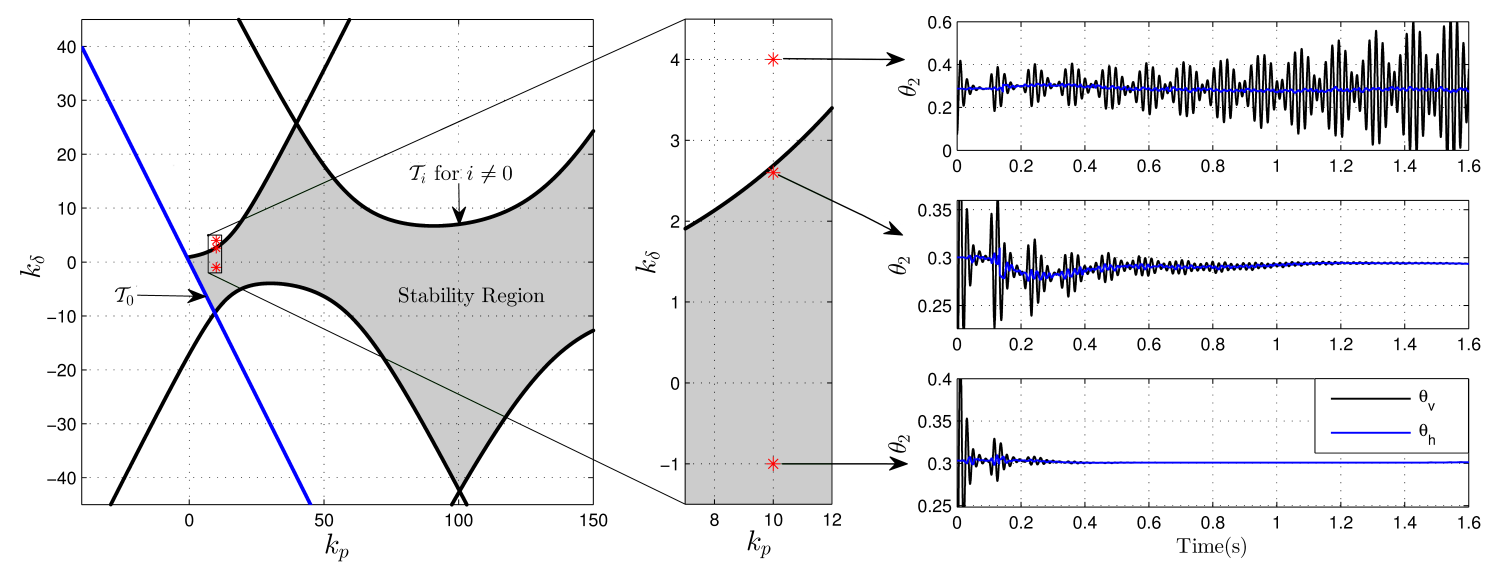

Figure 11. Stability regions VS Experimental behaviour for the joint two
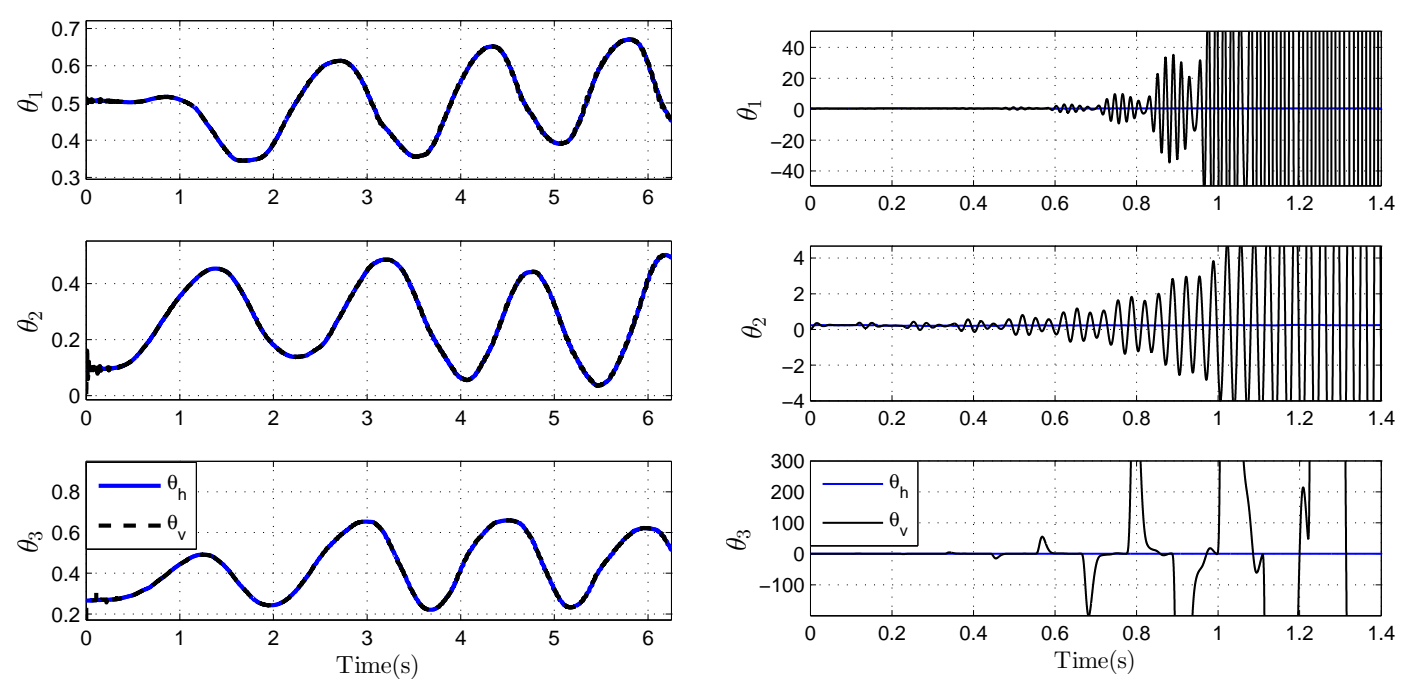

(a) Stable response

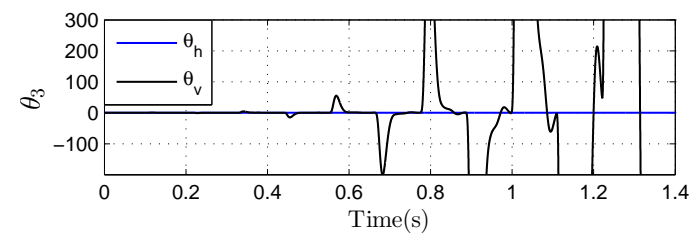

(b) Unstable response

Figure 12. Total system response

The three joints system is tested by choosing the controller gains either in stable and unstable regions (for further details see (Hernández-Díez et al., 2016)). The system response is shown in Fig.12. On Fig.12(a) one observes that choosing the controller gains in the stability region leads to a stable response with a good bilateral position tracking between the haptic and the virtual device. Fig.12(b) shows that choosing the controller gains in an unstable region produces an unstable 

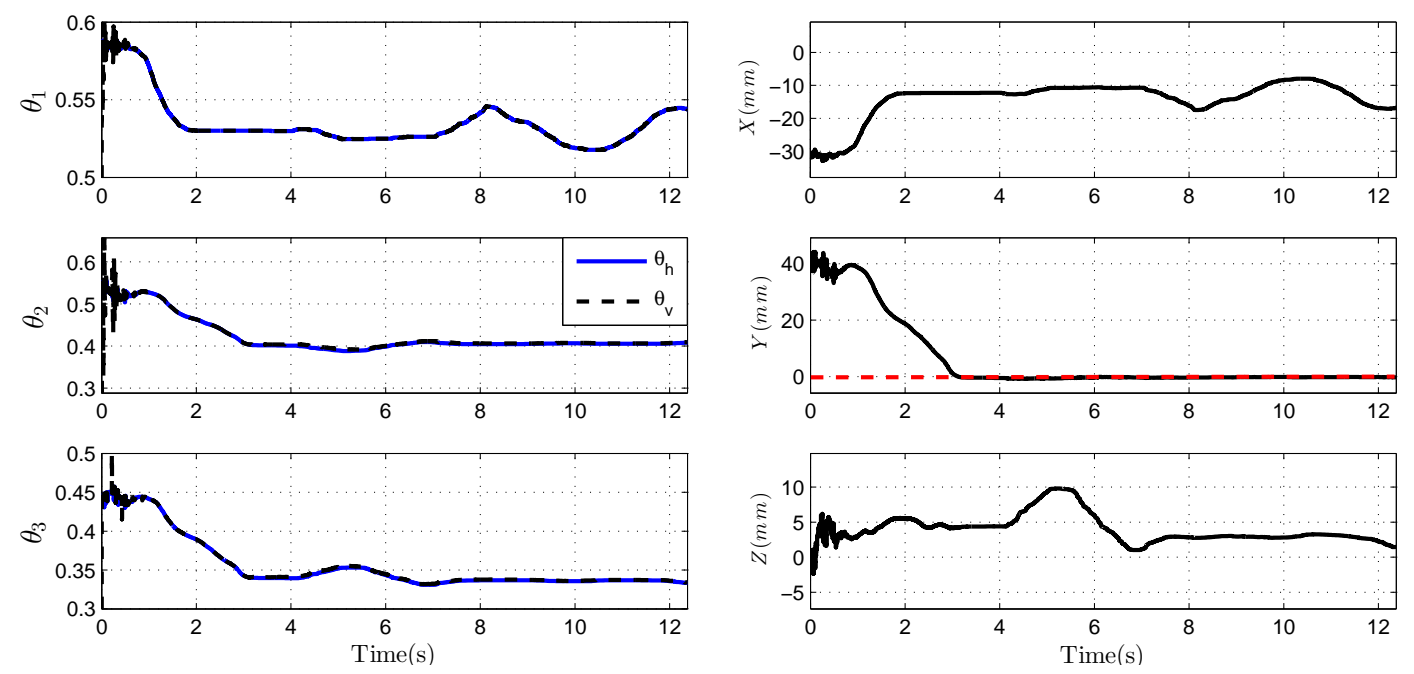

(a) Joints

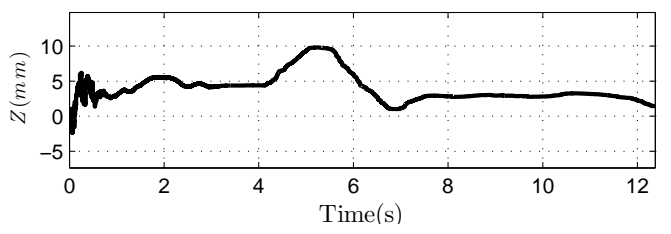

(b) Final effector position

Figure 13. Total system response perceiving a wall

system response, thus no tracking between both devices is achieved.

Finally, a virtual wall was implemented in the $X-Z$ plane applying a force $F_{y}$ normal to the $X-Z$ plane in the increasing direction of the $Y$ axis. The behavior of the wall is modeled as a simple spring with a Hooke constant $\boldsymbol{k}_{h}$ using the error between the position of the final effector of the haptic device in the $Y$ axis $Y_{f}$, and a fixed value $Y_{w}$, which is the location of the virtual wall on the $Y$ axis. This implementation is described by the following equation:

$$
F_{y}\left(Y_{f}\right)=\left\{\begin{array}{lll}
0 & \text { if } & Y_{f}>Y_{w}, \\
K_{h}\left(Y_{w}-Y_{f}\right) & \text { if } & Y_{f} \leqq Y_{w} .
\end{array}\right.
$$

In the implementation the correspondent torques perturbations $T_{e}$ for each joint produced by the virtual environment are obtained by using the following equation:

$$
T=J^{T} F,
$$

where $T$ contains each correspondent $T_{e}$ for each joint, $F=\left(0, F_{y}, 0\right)^{T}$ represents the forces vector applied by the virtual environment modeled as a virtual wall and $J$ is the jacobian matrix (see, for instance (Spong \& Vidyasagar, 1989)), obtained directly from the direct kinematic model studied by (Tavakoli et al., 2003). The results using $\boldsymbol{k}_{h}=1$ and $Y_{w}=0$ are shown in Figs. 13(a) and 13(b), where it is easy to observe how the haptic device moves freely until second three where the virtual wall restricts the movement along the $Y$ axis producing the human operator perception of the virtual wall.

\section{Concluding remarks}

A methodology for the design of a $P-\delta$ controllers applied to the stabilization of general LTI-SISO systems is presented. In addition, the behavior of the roots of the characteristic equation, as the controller gains are varied is analyzed in detail. A number of technical results concerning even open-loop transfer functions, multiple roots, and a new approach to the characterization of the stability index completes existing results on the construction of stability charts. The results go 
beyond the stabilization problem, particulary, the closed-loop performance analysis and the study of controllers fragility are addressed.

The design methodology can be applied and developed straightforwardly, showing that the presented results are easy to implement. Several examples covering all possible cases supports such statement. Finally, an experimental case study concerning the stabilization of a haptic-virtual system, illustrates the effectiveness of the $P-\delta$ controller.

\section{References}

Abdallah, C., Dorato, P., Benitez-Read, J., \& Byrne, R. (1993). Delayed positive feedback can stabilize oscillatory systems. In Proc. American Contr. Conf. (pp. 3106-3107).

Ackermann, J. (2002). Robust control. The parameter space approach. London: Springer.

Altmann, W., \& Macdonald, D. (2005). Practical Process Control for Engineers and Technicians. Elsevier/Newnes.

Aström, K. J., \& Hägglund, T. (1995). PID Controllers: Theory, Design, and Tuning (2nd ed.). Instrument Society of America, Research Triangle Park, NC.

Aström, K. J., \& Hägglund, T. (2001). The Future of PID Control. Chem. Eng. Progress, 9(11), $1163-1175$.

Bhattacharyya, S., Chapellat, H., \& Keel, L. (1995). Robust control. The parametric approach. Prentice Hall.

Cullen, C. G. (1990). Matrices and Linear Transformations: Second Edition (2nd ed.). Dover Publications.

Datko, R. (1978). A Procedure for Determination of the Exponential Stability of Certain Differential-Difference Equations. Quarterly of Applied Mathematics, 36(3), 279-292.

Gryazina, E. N. (2004). The D-Decomposition Theory. Automation and Remote Control, 65(12), $1872-1884$.

Gryazina, E. N., Polyak, B. T., \& Tremba, A. A. (2008). D-decomposition technique state-of-theart. Automation and Remote Control, 69(12), 1991-2026.

Guggenheimer, H. (1977). Differential Geometry. New York: Dover Publications.

Hale, J. K., \& Verduyn Lunel, S. M. (1993). Introduction to Functional Differential Equations (Vol. 99). New York: Springer-Verlag.

Hernández-Díez, J. E., González-Galván, E. J., Méndez-Barrios, C. F., Niculescu, S. I., LoredoFlores, A., \& Hernández-Molinar, R. I. (2016). A Bilateral Control Scheme of A HapticVirtual System Using Proportional-Delayed Controllers. AMRob Journal, Robotics: Theory and Applications, 49(2), 1-7.

Kharitonov, V., Niculescu, S.-I., Moreno, J., \& Michiels, W. (2005). Static output feedback stabilization: Necessary conditions for multiple delay controllers. IEEE Transactions On Automatic Control, 50(1), 82-86.

Krall, A. M. (1964). On the Real Parts of Zeros of Exponential Polynomials. Bulletin of the American Mathematical Society, 70(2), 291-292.

Levinson, N., \& Redheffer, R. (1970). Complex Variables. San Francisco, CA: Holden-Day, Inc.

Mazenc, F., Mondié, S., \& Niculescu, S.-I. (2003). Global asymptotic stabilization for chains of integrators with a delay in the input. IEEE Transactions on Automatic Control, 48(1), $57-63$.

Méndez-Barrios, C.-F. (2011). Low-Order Controllers for Time-Delay Systems : an Analytical Approach (Thesis). Université Paris Sud-XI.

Méndez-Barrios, C.-F., Niculescu, S.-I., Morărescu, I.-C., \& Gu, K. (2008). On the Fragility of PI Controllers for Time-Delay SISO Systems. In 16th Mediterranean Conference on Control and Automation (pp. 529-534). Ajaccio, France. 
Michiels, W., \& Niculescu, S.-I. (2014). Stability, Control, and Computation for Time-Delay Systems. An Eigenvalue-Based Approach. Philadelphia: SIAM.

Morărescu, I.-C., Méndez-Barrios, C.-F., Niculescu, S.-I., \& Gu, K. (2011). Stability Crossing Boundaries and Fragility Characterization of PID Controllers for SISO Systems with I/O Delays. In American Control Conference (ACC'11) (pp. 4988-4993). San Francisco, USA.

Morărescu, I.-C., \& Niculescu, S.-I. (2007). Stability crossing curves of SISO systems controlled by delayed output feedback. Dynamics of Continuous, Discrete and Impulsive Systems, 14(5), 659-678.

Neimark, J. (1949). D-subdivisions and spaces of quasi-polynomials. Prikl. Math. Mech., 13, 349-380.

Niculescu, S. I. (2001). Delay Effects on Stability: A Robust Control Approach. Heidelberg: Springer.

Niculescu, S.-I., \& Michiels, W. (2004). Stabilizing a chain of integrators using multiple delays. IEEE Trans. Aut. Control, 49(5), 802-807.

Niculescu, S.-I., Michiels, W., Gu, K., \& Abdallah, C. (2010). Delay effects on output feedback control of dynamical systems. In F. Atay (Ed.), Complex Time-Delay Systems (pp. 63-84). Berlin: Springer-Verlag.

O'Dwyer, A. (2009). Handbook of PI and PID Controller Tuning Rules (3rd ed.). London: Imperial College Press (ICP).

Ramírez, A., Mondié, S., Garrido, R., \& Sipahi, R. (2016). Design of Proportional-IntegralRetarded (PIR) Controllers for Second-Order LTI Systems. IEEE Transactions On Automatic Control, 61(6), 1688-1693.

Silva, G., Datta, A., \& Bhattacharrya, S. (2005). PID Controllers for Time Delay Systems. Boston: Birkhäuser.

Sipahi, R., Niculescu, S. I., Abdallah, C., Chaouki, T., Michiels, W., \& Gu, K. (2011). Stability and Stabilization of Systems with Time Delay: Limitations and Opportunities. IEEE Control Systems Magazine, 31(1), 38-65.

Smuts, J. F. (2011). Process Control for Practitioners. OptiControls Inc.

Spong, M. W., \& Vidyasagar, M. (1989). Robot Dynamics and Control. John Wiley \& Sons, Inc.

Suh, H., \& Bien, Z. (1980). Use of Time-Delay Actions in the Controller Design. IEEE Transactions On Automatic Control, 25(3), 600-603.

Tavakoli, M., Patel, R. V., Moallem, M., \& Aziminejad, A. (2003). Haptics for Teleoperated Surgical Robotic Systems. World Scientific Publishing Company.

Villafuerte, R., Mondié, S., \& Garrido, R. (2013). Tuning of Proportional Retarded Controllers: Theory and Experiments. IEEE Transactions On Control Systems Technology, 21(3), 983990. 